\title{
Experimental study of rf pulsed heating on oxygen free electronic copper
}

\author{
David P. Pritzkau* and Robert H. Siemann ${ }^{\dagger}$ \\ Stanford Linear Accelerator Center, Stanford University, Stanford, California 94309
}

(Received 4 September 2002; published 21 November 2002)

\begin{abstract}
When the thermal stresses induced by rf pulsed heating are larger than the elastic limit, microcracks and surface roughening will occur due to cyclic fatigue. Therefore, pulsed heating limits the maximum surface magnetic field and through it the maximum achievable accelerating gradient. An experiment using circularly cylindrical cavities operating in the $\mathrm{TE}_{011}$ mode at a resonant frequency of $11.424 \mathrm{GHz}$ was designed to study pulsed heating on oxygen free electronic (OFE) copper. An $X$-band klystron delivered up to $10 \mathrm{MW}$ to the cavities in $1.5 \mu$ s pulses at $60 \mathrm{~Hz}$ repetition rate. One run was executed at a temperature rise of $120 \mathrm{~K}$ for $56 \times 10^{6}$ pulses. Cracks at grain boundaries, slip bands, and cracks associated with these slip bands were observed. The second run consisted of $86 \times 10^{6}$ pulses with a temperature rise of $82 \mathrm{~K}$, and cracks at grain boundaries and slip bands were seen. Additional information can be derived from the power-coupling iris, and we conclude that a pulsed temperature rise of $250 \mathrm{~K}$ for several million pulses leads to destruction of copper. These results can be applied to any mode of any OFE copper cavity.
\end{abstract}

DOI: 10.1103/PhysRevSTAB.5.112002

PACS numbers: 84.40.-x, 29.17.+w

\section{INTRODUCTION}

rf pulsed heating is a process by which metals are heated by the eddy currents created by a pulsed highpower rf source. Stress is induced on the metal surface when the heating occurs faster than the material has time to expand, which is governed by the speed of sound in the material. Such material will be prone to cyclic fatigue in which damage in the form of surface roughening and microcracks will occur over time if the induced stress is greater than the yield strength of the material.

\section{A. Relevance to accelerator physics}

Accelerating gradients in room temperature linear accelerators are limited by rf breakdown, dark current trapping, and pulsed heating. As one example, Wilson has taken the geometry of the Next Linear Collider traveling wave structure [1] and developed parametrizations for these limits with wavelength $(\lambda)$. They are

$$
\begin{aligned}
G_{\text {break }} & =\frac{1.1 \mathrm{GeV} / \mathrm{m}}{[\lambda(\mathrm{cm})]^{7 / 8}}, \\
G_{\text {trap }} & =\frac{1.6 \mathrm{MeV}}{\lambda}, \\
G_{\text {pulse }} & =(28 \mathrm{MeV} / \mathrm{m}) \frac{\Delta T^{1 / 2}}{[\lambda(\mathrm{mm})]^{1 / 8}} .
\end{aligned}
$$

A plot of these limits (Fig. 1) shows that pulsed heating could become the most restrictive phenomenon at short wavelength. Recent experiments have shown that breakdown is a multifaceted phenomenon [3,4], and gradient limits are not as simple as implied by Eqs. (1). Underlying physical phenomena are complex, material properties are important, and details of the structure geometry can matter because local regions of high field or high pulsed heating may determine the overall behavior.
The experiment reported in this paper was designed to study the effects of pulsed heating on OFE copper, the material most commonly used in normal conducting accelerator structures. (OFE copper is oxygen free electronic copper. It is $\mathrm{C} 10100$ in the copper unified numbering system.) The experiment has been performed with a cavity geometry and mode where pulsed heating was the dominant effect. The results are observations of pulsed heating effects and an upper limit on the maximum tolerable temperature rise. The limit can be applied to any cavity and any mode by calculating the electromagnetic fields and pulsed temperature rise throughout. If the

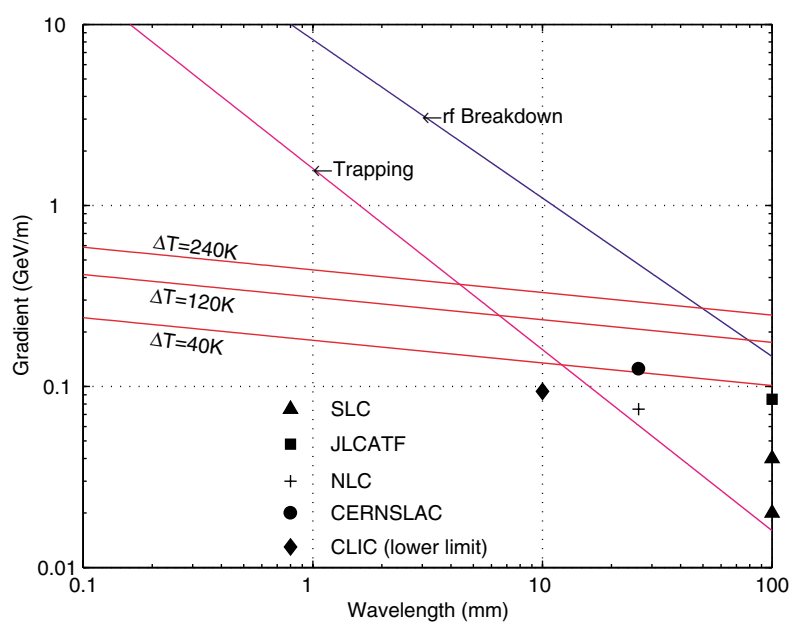

FIG. 1. (Color) Gradient limits due to rf breakdown, dark current trapping, and pulsed heating. The data points represent gradient achieved with reasonable dark current [2]. The gradient limit for pulsed heating assumes a damage threshold of $\Delta T=40 \mathrm{~K}$ and is scaled from parameters of the Next Linear Collider (NLC) assuming the filling time scales as $T_{p} \sim \lambda^{3 / 2}$. 
temperature rise in part of the structure exceeds the maximum tolerable amount, the structure will have a limited gradient and/or, as the experiment shows, a limited lifetime.

\section{B. Thermal fatigue}

Copper was studied in this experiment because of the preponderance of use in rf cavities. For copper, the boundary between elastic and plastic deformation is reached gradually with changes in stress or strain. In the region of elastic deformation, no permanent deformation will occur when stress is removed and no damage occurs. Under plastic deformation, when the applied stress or strain is beyond the yield point, permanent changes will be left in the material. Microscopically, atoms at different planes in the crystal lattice will slip past each other causing vacancies and different configurations. If the metal is constantly stressed in the plastic region, these microscopic changes will accumulate as damage. This damage, known as cyclic fatigue, usually occurs at the surface because of the discontinuity of the crystal lattice. It evolves in the form of slip planes and microcracks on the surface.

The stress or strain necessary to cause damage by cyclic fatigue is much less than that required to break the material under static deformation. However, damage from cyclic fatigue occurs after many cycles of stress or strain. It generally occurs in three stages. The first stage is cyclic hardening of the material, in which the metal becomes stronger. The stress necessary to cause a certain amount of strain increases. This stage occurs within a few percent of the cycles to failure.

The second stage is the crack nucleation stage. In this stage, slip and cracks begin to initiate in the metal. They usually occur on the free surface of the metal and are most likely due to localized concentrations of plastic strain or imperfections in the crystal lattice.

The third stage is crack propagation. In this stage, fracture mechanics is used to describe the propagation of the cracks on the surface. Normally, the total lifetime of a metal is defined by the number of cycles it takes for cracks to nucleate plus the number of cycles it takes cracks to reach a particular length. Crack propagation is usually responsible for mechanical failure.

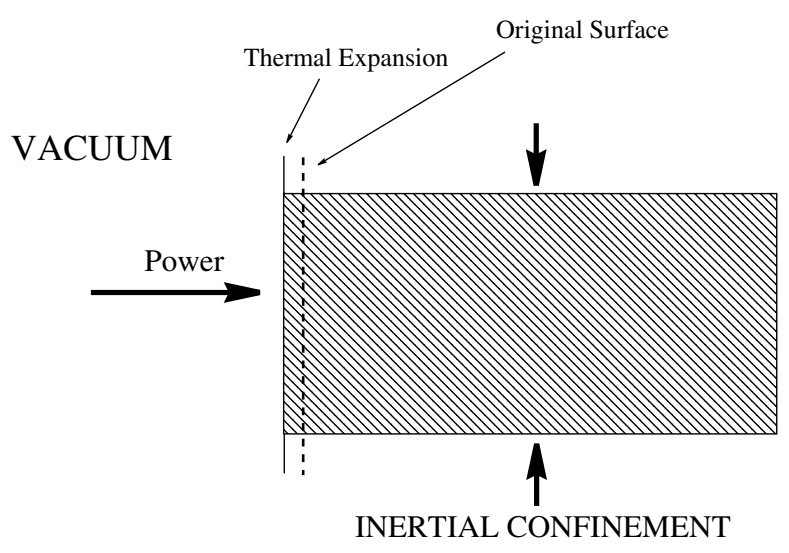

FIG. 2. Metal surface being heated with a fast high-power source [5]. The surface itself expands normally into the vacuum.

In the study of rf pulsed heating, we are more interested in the changes of electrical performance, so the nucleation of cracks is more relevant. Cracks on the surface will increase the surface resistivity of copper and degrade the cavity $Q$.

Thermal fatigue is cyclic fatigue caused by repetitive heating and cooling. Eddy currents that are developed from tangential magnetic fields on the surface will heat the surface. A simple example is shown in Fig. 2 where a fast high-power source heats the surface [5]. The heating happens quickly enough that the bulk of the metal is inertially confined and cannot expand transversely. Inertial confinement is the mechanical constraint that causes stress to occur on the surface. Using static linear elasticity with the assumptions that the transverse strain and normal stress are zero, the temperature rise at which the stress on the surface exceeds the yield stress $\sigma_{y}$ is [5]

$$
\Delta T_{y}=\frac{(1-\nu) \sigma_{y}}{E \alpha}
$$

where $\nu$ is Poisson's ratio, $E$ is the elastic modulus, and $\alpha$ is the coefficient of linear thermal expansion.

For temperature rises above $\Delta T_{y}$, plastic deformation and damage will occur. However, due to hysteresis, the stress must exceed the yield stress in both tension and compression in order for damage to continuously occur.

TABLE I. Parameters of fully annealed OFE copper at room temperature.

\begin{tabular}{lc}
\hline \hline$\rho$ (density) & $8.95 \times 10^{3} \mathrm{~kg} / \mathrm{m}^{3}[6]$ \\
$c_{\varepsilon}$ (specific heat at constant strain) & $385 \mathrm{~J} / \mathrm{kg} \mathrm{K} \mathrm{[6]}$ \\
$k$ (thermal conductivity) & $390 \mathrm{~W} / \mathrm{m} \mathrm{K} \mathrm{K6]}^{-5}$ \\
$\alpha$ (coefficient of linear thermal expansion) & $1.65 \times 10^{-5} \mathrm{~K}^{-1}[6]$ \\
$\rho_{\text {res }}$ (electrical resistivity) & $1.7 \times 10^{-8} \Omega \mathrm{m} \mathrm{[6]}$ \\
$E$ (elastic modulus) & $123 \mathrm{GPa}[7]$ \\
$\nu$ (Poisson's ratio) & $0.345[7]$ \\
$\sigma_{y}$ (yield stress) & $62 \mathrm{MPa} \mathrm{[5]}$ \\
\hline \hline
\end{tabular}


Hence, the threshold temperature for damage due to pulsed heating occurs above $2 \Delta T_{y}$ [5]. Using the numbers given in Table I for fully annealed OFE copper, the threshold temperature rise is $40 \mathrm{~K}$. Uncertainties associated with the mechanical properties of fully annealed OFE copper give significant uncertainty in this temperature threshold. Different surface preparations will affect the lifetime of the material [8]. Also, other sources have identified a safe threshold of a $110 \mathrm{~K}$ temperature rise for pulsed heating from electron flux $[9,10]$. However, the preparation of the surface in $[9,10]$ was not specified and could be much different than that used in the experiments described in this paper.

\section{EXPERIMENTAL SETUP}

We used a $\mathrm{TE}_{011}$ mode in a circular cylindrical cavity with the length and radius chosen to maximize the temperature rise on the end caps of the cylinders for two reasons. First, the purely azimuthal electric field in the cavity will minimize the chance of rf field breakdown on any surface in the cavity. This will isolate the effects of pulsed heating damage to the cavity surface. Second, the $\mathrm{TE}_{011}$ mode does not require physical contact between the end caps and the cylindrical sidewall. This simplifies removal and analysis of the damaged end cap surfaces. It would also allow reuse of the main body of the cavity with end caps with different materials or surface preparations, but this was not done. Not only are the end caps easily removable, they allow for simple tuning of the cavity by adjusting its axial length.

The $\mathrm{TE}_{011}$ mode is excited by high-power rf pulses from the available $50 \mathrm{MW}, 1.5 \mu \mathrm{s}$ pulse, $11.424 \mathrm{GHz}$ ( $X$-band) klystrons at SLAC. Because of the high $Q$ of the cavity, long pulse lengths are desired, so no pulse compression systems are used. At a repetition rate of $60 \mathrm{~Hz}$, the surface heats up on every rf pulse then cools back down to the ambient temperature by the time the next rf pulse begins.

During the experiment, it would be ideal to measure the pulsed temperature rise on the cavity surface as well as the $\mathrm{rf}$ properties of the cavity. Since we are most interested in the effects of pulsed heating on these $\mathrm{rf}$ properties, we performed some measurements by parasitically exciting a low-power steady-state $\mathrm{TE}_{012}$ mode. As the surface heats up, the $Q$ will decrease due to the increase of the surface resistivity with temperature. The coupling to the $\mathrm{TE}_{012}$ mode will dynamically change with the rf pulse. By measuring the change in reflected power from the coupling to the $\mathrm{TE}_{012}$ mode, the temperature rise of the surface of the cavity can be extracted.

\section{A. Cavity design}

The dimensions of the cavity were optimized such that the maximum heating occurs on the cavity end caps. It is also desirable to minimize the heating on the cylindrical sidewall. Since the lifetime of copper varies as a power law with the applied stress $[8,11]$, the cavity may be reused several times with different end caps before it becomes too damaged.

On the end caps, the tangential magnetic field is purely radial and varies as $J_{1}\left(p_{11} r / R\right)$, where $R$ is the radius of the end cap and $p_{11}$ is the first zero of $J_{1}(x)$. The local power dissipated on the surface varies as the square of the magnetic field, hence the temperature rise on the surface of the end caps will appear as zones similar to tree rings. The maximum, which occurs at $48 \%$ of the cavity radius, will be referred to as the maximum temperature rise. Figure 3 shows the maximum normalized temperature rise of the cavity end caps and sidewall for a fixed resonant frequency of $11.424 \mathrm{GHz}$ with various cavity lengths. The maximum temperature rise on the sidewall occurs in the center $(d / 2)$. The most appropriate cavity length is $d=1.90 \mathrm{~cm}$.

At this length, the optimum coupling to the $\mathrm{TE}_{011}$ mode is $\beta=1.28$. The maximum temperature rise slowly varies with $\beta$ about this optimum value which increases the tolerances for machining and simulation.

The input power is coupled to the cavity through the magnetic field developed in a $\mathrm{TE}_{10}$ mode inside a waveguide mounted on the cylindrical sidewall centered along the cavity's axial length. The input coupler is a standard WR-90 waveguide with a circular aperture cut into the middle of its cross section. The coupling coefficient $\beta$ determines the aperture size used to couple from the input waveguide to the cavity. The correct aperture size was determined through computer simulation using the finitedifference codes MAFIA [12] and GDFIDL [13]. Because of a nonperfect surface finish from machining, we assumed

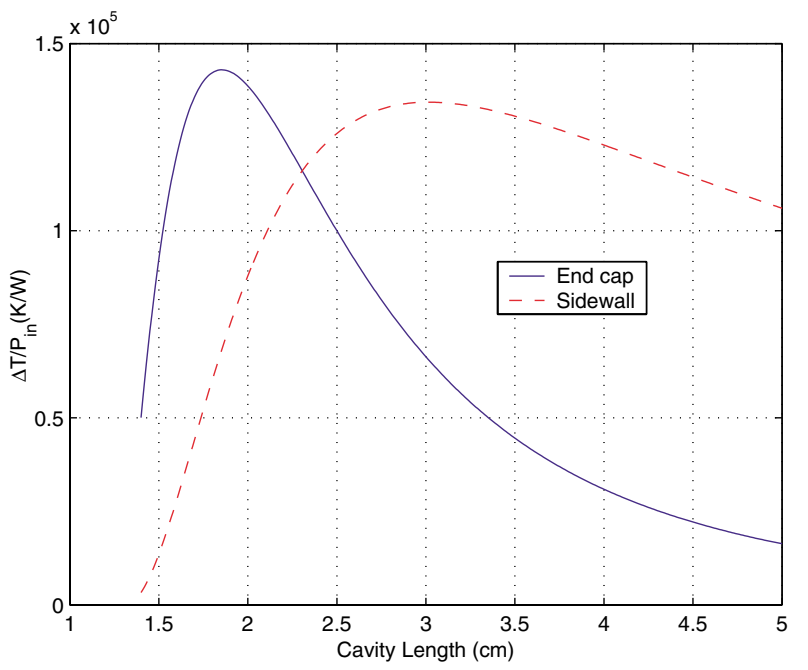

FIG. 3. (Color) Maximum temperature rises on cavity end caps and sidewall normalized to input power as a function of cavity length $d$ at a fixed resonant frequency of $11.424 \mathrm{GHz}$. This plot does not include consideration of temperature rise at the coupling iris. 
TABLE II. Cavity design parameters for the $\mathrm{TE}_{011}$ mode.

\begin{tabular}{lc}
\hline \hline$f_{\text {res }}$ (resonant frequency) & $11.424 \mathrm{GHz}$ \\
$d$ (axial length) & $1.90 \mathrm{~cm}$ \\
$R$ (radius) & $2.2075 \mathrm{~cm}$ \\
$Q_{0}$ (unloaded $\left.Q\right)$ & 21900 \\
Estimated $Q_{0}($ unloaded $Q$ with estimated degradation) & 19710 \\
$Q_{\text {ext }}$ (external $\left.Q\right)$ & 15400 \\
$Q_{L}$ (loaded $\left.Q\right)$ & 8650 \\
$\beta$ (coupling coefficient) & 1.28 \\
$R_{a}$ (radius of coupling aperture) & $3.75 \mathrm{~mm}$ \\
$d_{a}$ (thickness of coupling aperture) & $1.88 \mathrm{~mm}$ \\
\hline \hline
\end{tabular}

a $10 \%$ degradation in the unloaded $Q$ of the cavity to determine the aperture size. The design parameters for the $\mathrm{TE}_{011}$ mode are given in Table II.

The parameters for the $\mathrm{TE}_{012}$ mode, except for the coupling, are fixed from the cavity dimensions. This mode is also excited through a waveguide that is centered along the cylindrical sidewall at $1 / 4$ the cavity's axial length, a position where the magnetic field of the $\mathrm{TE}_{012}$ mode is at its maximum. The width of the input coupler is that of a WR-42 waveguide (0.42 in.) so that its cutoff frequency is above $11.424 \mathrm{GHz}$. The width was tapered to $0.622 \mathrm{in}$. after a length of $10.4 \mathrm{~cm}$ to make use of available WR-62 vacuum windows. The length was chosen such that the power at $11.424 \mathrm{GHz}$ would be attenuated by over $150 \mathrm{~dB}$ to avoid damaging the measurement electronics. The input coupler is designed to critically couple into the $\mathrm{TE}_{012}$ mode so that the steady-state reflected power is nominally zero when there is no temperature rise in the cavity. The design parameters for the $\mathrm{TE}_{012}$ mode are shown in Table III. A $10 \%$ degradation in $Q_{0}$ was similarly assumed.

A picture of the test cavity with one end cap placed outside is shown in Fig. 4. An assembly schematic with both end caps inserted into the cavity is shown in Fig. 5.

A close-up schematic of an end cap assembly is shown in Fig. 6. The bellows and differential-screw assembly for each end cap allow fine-tuning of the cavity resonant frequency. The copper end cap is mounted onto a stainless-steel piston assembly in order to facilitate the reuse of the assembly for future end caps. A detailed assembly procedure for the end cap is given in Table IV. The preparation of the end cap surface will impact the measured lifetime of the material [8]. The steps and chemicals used for vacuum cleaning of the copper end cap surfaces mentioned in step 6 of Table IV are detailed in Table V.

A spiral channel is cut into the back of each end cap for water cooling to remove the average heat that is

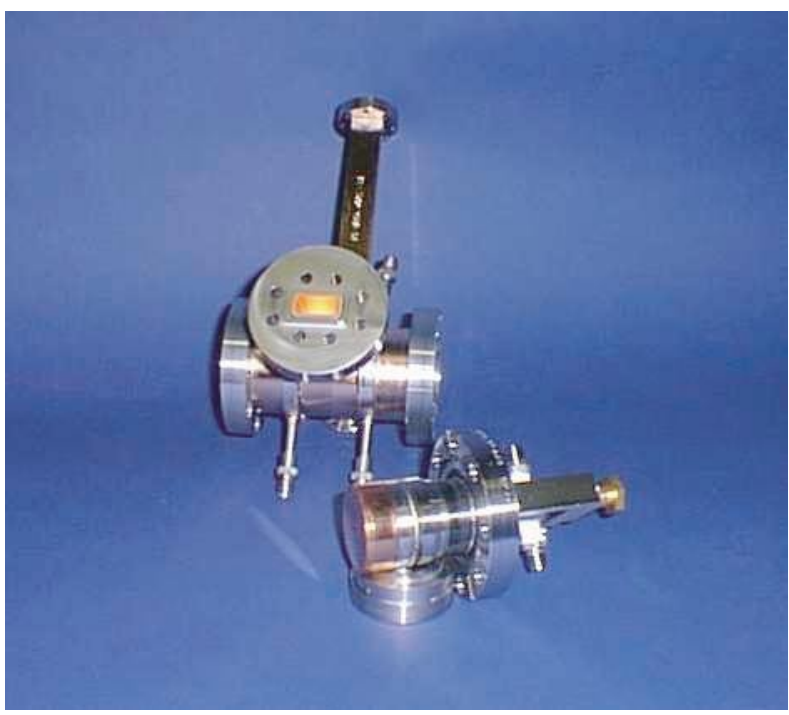

FIG. 4. (Color) A picture of the cavity body with one end cap shown on the outside. The water-cooling pipes can be seen on the bottom of the cavity body.

TABLE III. Cavity design parameters for the $\mathrm{TE}_{012}$ mode.

\begin{tabular}{lc}
\hline \hline$f_{\text {res }}$ (resonant frequency) & $17.820 \mathrm{GHz}$ \\
$Q_{0}$ (unloaded $\left.Q\right)$ & 21960 \\
Estimated $Q_{0}($ unloaded $Q$ with estimated degradation) & 19760 \\
$Q_{\text {ext }}($ external $Q)$ & 19760 \\
$Q_{L}$ (loaded $\left.Q\right)$ & 9880 \\
$\beta$ (coupling coefficient) & 1.0 \\
$R_{a}$ (radius of coupling aperture) & $2.85 \mathrm{~mm}$ \\
$d_{a}$ (thickness of coupling aperture) & $1.034 \mathrm{~mm}$ \\
\hline \hline
\end{tabular}



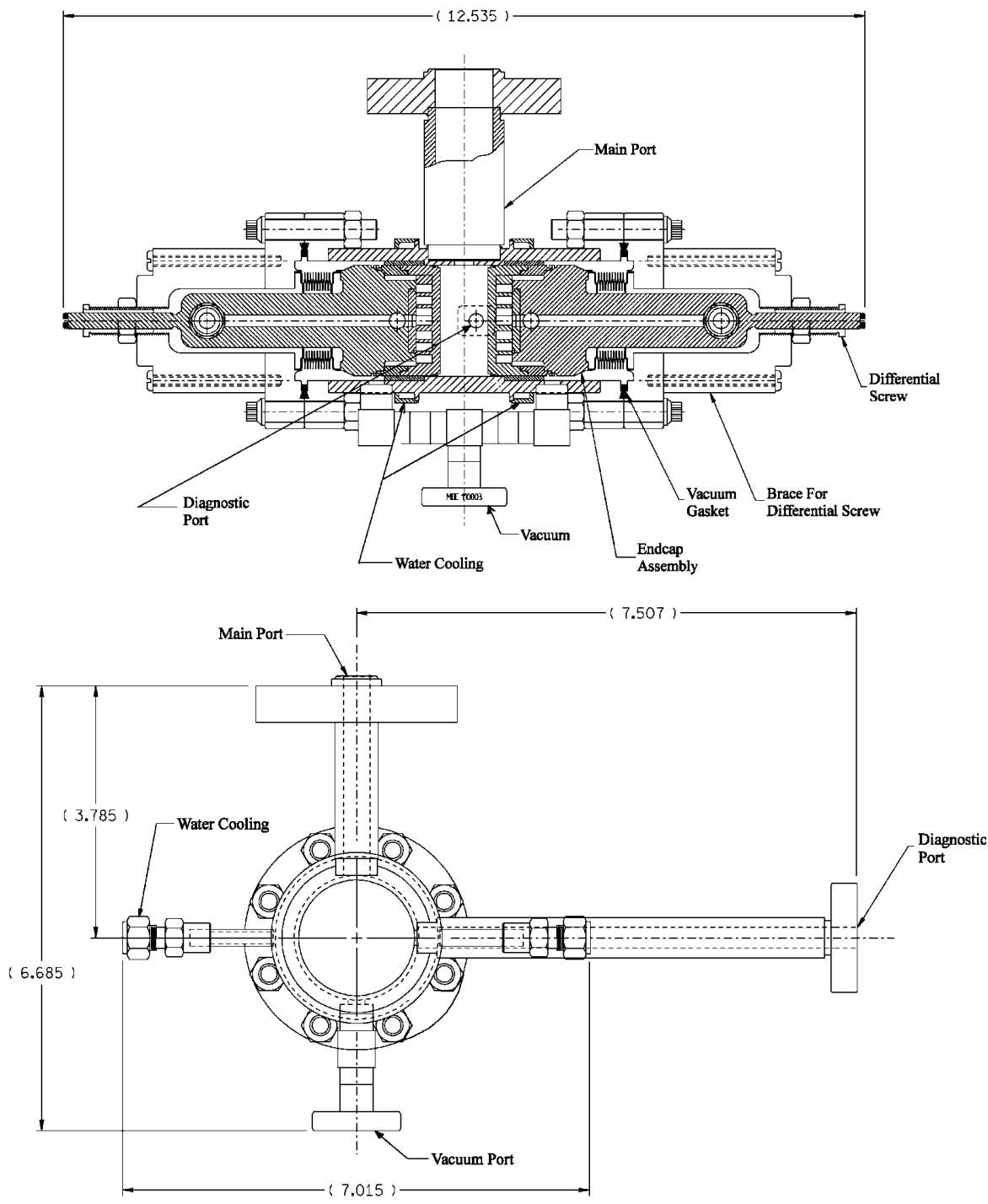

FIG. 5. Assembly schematic of cavity with end caps. The sideview of the cavity is shown on the bottom. All dimensions are in inches.

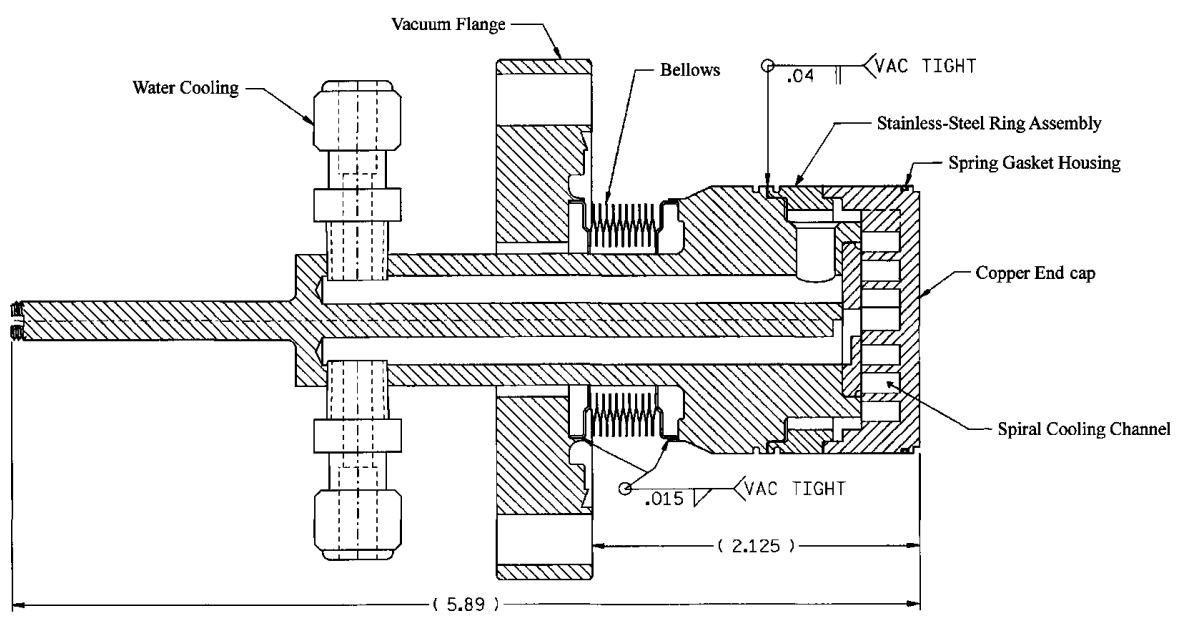

FIG. 6. Assembly schematic of end cap and piston. All dimensions are in inches. 
TABLE IV. Piston assembly and copper end cap surface preparation.

\begin{tabular}{cc}
\hline \hline STEP & DESCRIPTION \\
\hline 1 & Cooling channel and grooves machined on copper end cap \\
2 & Surface of copper end cap machined to class-16 finish \\
3 & Copper end cap brazed to stainless-steel ring \\
4 & Stainless-steel ring assembly machined to correct dimensions \\
5 & Surface of copper end cap diamond fly-cut to mirror finish \\
6 & Surface of copper end cap chemically etched for 5 sec for vacuum cleaning \\
7 & Stainless-steel ring assembly welded to stainless-steel piston \\
8 & rf spring gaskets placed in rf groove around circumference of copper end cap \\
9 & Piston assemblies inserted into cavities and placed under vacuum \\
\hline \hline
\end{tabular}

TABLE V. Procedure for vacuum cleaning of end cap surface.

\begin{tabular}{cc}
\hline \hline STEP & DESCRIPTION \\
\hline 1 & Vapor degrease in $1,1,1$ trichloroethane [14] for $5 \mathrm{~min}$ \\
2 & Alkaline soak clean using Enbond Q527 [15] for 5 min at $180^{\circ} \mathrm{F}$ \\
3 & Cold tap water rinse for 2 min \\
4 & Immerse in $50 \%$ hydrochloric acid at room temperature for $1 \mathrm{~min}$ \\
5 & Cold tap water rinse for $1 \mathrm{~min}$ \\
6 & Immerse in the following solution for 5 sec at room temperature: \\
& Phosphoric acid, $75 \% 21$ gal \\
& Nitric acid, $42^{\circ}$ Baumé 7 gal \\
7 & Acetic acid, Glacial 2 gal \\
8 & Hydrochloric acid 19.2 fluid oz \\
9 & Cold tap water rinse for minimum of 2 min \\
10 & Cold tap water rinse for 1 min \\
11 & Cold deionized water rinse for $1 \mathrm{~min}$ \\
12 & Hot deionized water rinse for $30 \mathrm{sec}$ \\
13 & Blow dry with dry nitrogen blast \\
14 & Dry in air oven at $150^{\circ} \mathrm{F}$ \\
\hline \hline
\end{tabular}

dissipated. This prevents the cavity's resonant frequency from drifting due to thermal expansion. Water-cooling pipes were also added around the circumference of the cavity on both sides of the input coupler as shown in Figs. 4 and 5.

Two grooves are cut along the outer radius of the end caps as shown in Fig. 7. One groove is used to remove the degeneracy between the $\mathrm{TE}_{011}$ mode and the $\mathrm{TM}_{111}$ mode as well as the degeneracy between the $\mathrm{TE}_{012}$ mode and

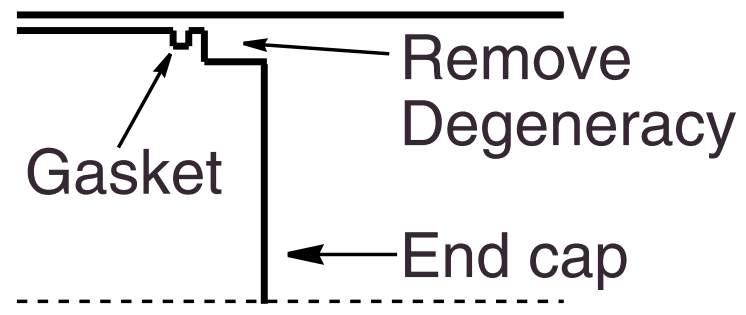

FIG. 7. Close-up of end cap with grooves. Not to scale. the $\mathrm{TM}_{112}$ mode. The TM modes require current to cross from the end cap to the cylindrical sidewall, hence their resonant frequencies are shifted by creating a gap. The gap between this groove and the sidewall is $1.1 \mathrm{~mm}$. The depth of the groove was initially set to $1.0 \mathrm{~mm}$. However, at this depth, there were interferences of other modes with the $\mathrm{TE}_{012}$ mode that prevented the temperature diagnostic from being used during the first high-power test. Using a cavity mock-up, the correct depth of the groove was empirically determined to be $2.5 \mathrm{~mm}$ to isolate the $\mathrm{TE}_{012}$ mode and this was used for the second high-power test.

The second groove houses a gold-plated stainless-steel spring gasket that becomes an rf short when compressed. The rf short prevents coaxial modes from being excited in the gap along the length of the piston and prevents $\mathrm{rf}$ breakdown from occurring in this gap.

Cold-test results demonstrated some errors in the meshing of the input coupler during simulation. The measured coupling coefficient to the $\mathrm{TE}_{011}$ mode was approximately 1.7 which reduced the temperature rise per watt by 
approximately $4 \%$ from the optimum value. As indicated earlier, the $\mathrm{TE}_{012}$ mode suffered from interference with other modes which was fixed for the second high-power test. The cold-test results also demonstrated leakage of power from the $\mathrm{TE}_{012}$ mode into the input coupler for the $\mathrm{TE}_{011}$ mode. Hence the cavity has to be treated as a twoport cavity for the $\mathrm{TE}_{012}$ mode during data analysis. The comparison of the rf parameters of each mode before and after the high-power tests will be given in Sec. IV. See [17] for a complete discussion of the cold-test results.

\section{B. Klystron setup}

Two different setups were used to conduct high-power tests with $X$-band klystrons. They are shown in Fig. 8. Each setup is required to protect the klystron by limiting the reflected power to a maximum of $5 \mathrm{MW}$. Fifty-five $\mathrm{dB}$ directional couplers were placed in front of each cavity to measure the forward and reflected power. The power measurements allow us to determine the coupling and $Q$ of each cavity.

In the setup for the first high-power test, two similar cavities were used simultaneously. By using an asymmetric magic tee, the reflected power from each cavity would go to the load. The advantage of this setup is that each cavity would receive the maximum input power possible since no high-power circulators exist. Also, four end caps can be tested simultaneously. The disadvantage is that the cavities have to be tuned to each other while the klystron is on. The differential screws did not offer a fine enough adjustment, and the cavities have to be tuned by adjusting water flow to the end caps. Also, the measurement of the loaded $Q$ of each cavity suffered from systematics that will be explained later.

From the experiences gained with the first high-power test, we changed to the second setup. This setup has the advantage of relaxing the necessity of fine-tuning the cavity. Normally, the only disadvantage is the amount of power that must be thrown away in order to protect the klystron. However, due to $\mathrm{rf}$ breakdown that was known to occur at the aperture at approximately 8.5 MW from the first test, this was not a hindrance.

It is not possible to reach the desired power level in the cavity immediately due to absorbed gases in copper. Normally at SLAC high-power components are first placed under vacuum then baked out at high temperature (at least $400{ }^{\circ} \mathrm{C}$ ) for a few days to remove gas. However, the rf spring gaskets used to prevent the excitation of coaxial modes along the pistons cannot be baked at such a high temperature. They may lose their elastic properties causing their effectiveness as rf shorts to deteriorate. Thus, the cavity body and the magic tee were baked out separately at high temperature because they were new and were not handled in a clean environment. However, the end caps did not go through a hightemperature bakeout. Afterwards, the components were assembled and attached to the klystron. After being placed under vacuum, the components were baked using heater tapes at $150{ }^{\circ} \mathrm{C}$ for 3 days. This procedure removes some absorbed gas on the surface.

The klystron power level must be increased slowly as the high-power fields in the cavity and other waveguide components pull more gas out of the surface. This is known as power conditioning. There are interlocks to shut down the klystron when there is too much gas in the system. If this is not done, damage may result to the components due to $\mathrm{rf}$ breakdown. Over time, the gas is slowly removed and the power may be increased. As will be discussed later, rf breakdown at the high-power coupling aperture limited the amount of power that could be put into the cavity. At this point, gas is released violently and the cavity cannot be conditioned any further in a reasonable amount of time.

\section{Diagnostic setup}

The forward and reflected $X$-band powers are measured with crystal detectors attached to $55 \mathrm{~dB}$ directional couplers. Additional attenuation is added to give appropriate power levels for the crystals, which had sensitivities between 500 to $700 \mathrm{mV} / \mathrm{mW}$. The forward power signal is digitized, binned by power level and counted to measure the number of high-power pulses accrued when temperature rise and $Q$ 's of the $\mathrm{TE}_{011}$ and $\mathrm{TE}_{012}$ modes are measured.

The diagnostic setup that measures the pulsed temperature rise and $Q$ of the $\mathrm{TE}_{012}$ mode is shown in Fig. 9. A cw low-power $\mathrm{rf}$ source excites the diagnostic mode. The source frequency is roughly $17.8 \mathrm{GHz}$ and is adjusted to minimize reflected power. A waveguide low-pass filter
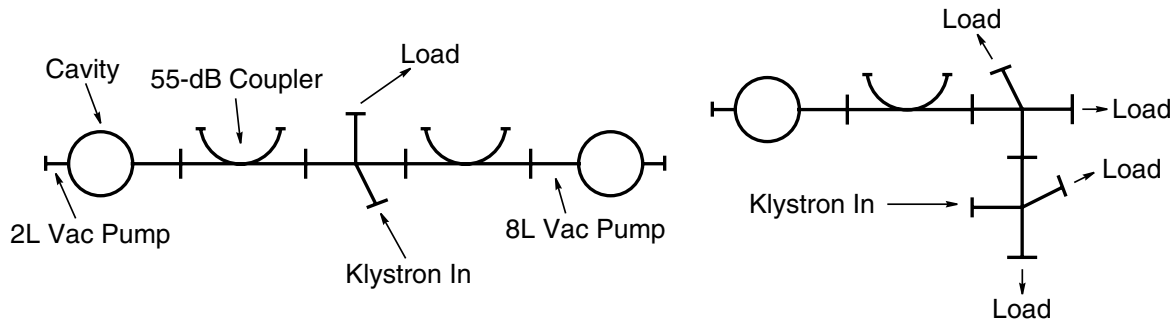

FIG. 8. Schematic of the klystron setups for the first and second high-power tests. 


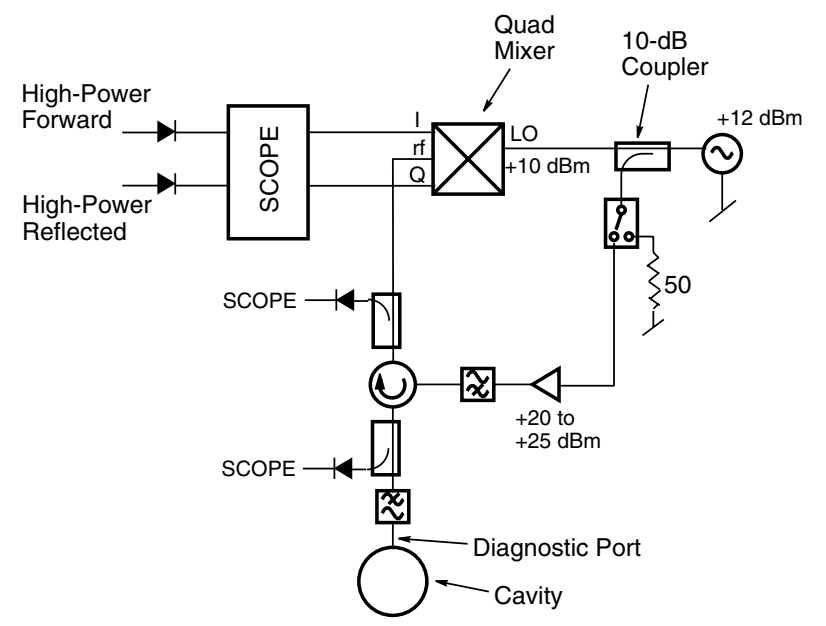

FIG. 9. Schematic of the diagnostic setup to measure pulsed temperature rise and cavity $Q$ of the $\mathrm{TE}_{012}$ mode.

protects the electronics from high-power klystron harmonics that are coupled out and are not cut off by the WR-42 hybrid waveguide. A circulator, with approximately $32 \mathrm{~dB}$ isolation at $18 \mathrm{GHz}$, isolates the reflected signal from the input signal, and directional couplers with crystal detectors are placed on either side of the circulator to measure forward and reflected powers. The steady-state reflection from the cavity with a coupling coefficient $\beta=0.7$ is $16 \mathrm{~dB}$ below the input power. There is $2 \mathrm{~dB}$ insertion loss in the circulator and directional coupler, so the reflected signal is $14 \mathrm{~dB}$ above the input power that leaks through the circulator. The reflected power increases during the pulsed heating, and the reflected signal can be measured without significant error caused by imperfect isolation. The amplitude and phase of the reflected cw signal will change when the cavity is heated from a high-power, $X$-band pulse. Those changes are measured with the quadrature mixer. The rf switch can be opened between high-power pulses when the temperature has returned to ambient, and the energy in the $\mathrm{TE}_{012}$ mode will drain from the cavity. The decay time and steady-state reflected power can be combined to give the coupling coefficient and unloaded $Q$. Changes in $Q_{0}$ indicate surface deterioration.

\section{THEORY}

The temperature rise on the surface of the cavity can be determined using the familiar heat conduction equation with knowledge of the electromagnetic fields in the cavity and the input power. In reality, the heat conduction equation is coupled to the elasticity equations because of the small amount of heat dissipation that occurs due to mechanical strain of the material. This coupling term is quite small in our case [17] and may be ignored.

\section{A. Heat conduction}

It is simplest to reduce the heat conduction equation to an integral using Green's functions. The surface heating is from the power dissipation that occurs from the eddy currents created from surface magnetic fields. Since the power dissipated per unit area on the surface is proportional to the square of the magnetic field, the heat generated per unit volume is

$$
g(z, t)=\frac{d P(z, t)}{d A d z}=\frac{d P(t)}{d A} \frac{2}{\delta} e^{-2 z / \delta}, \quad z \geq 0,
$$

where $\delta$ is the skin depth. For a one-dimensional infinite slab the temperature rise on the surface $z=0$ is

$$
\begin{aligned}
\Delta T(z=0, t)= & \frac{2}{\rho c_{\varepsilon}} \int_{0}^{t} d t^{\prime} \frac{d P\left(t^{\prime}\right)}{d A} \frac{1}{\delta} \exp \left[\frac{4 \alpha_{d}\left(t-t^{\prime}\right)}{\delta^{2}}\right] \\
& \times \operatorname{erfc}\left[\frac{2}{\delta} \sqrt{\alpha_{d}\left(t-t^{\prime}\right)}\right]
\end{aligned}
$$

where $\alpha_{d}=k / \rho c_{\varepsilon}$ is the thermal diffusivity.

For a square rf pulse of length $t_{p}$, the diffusion depth $D=\sqrt{\alpha_{d} t_{p}}$ characterizes the length into the surface of which the effect of the heat source decays on the order of $1 / e$ at time $t_{p}$. If the diffusion depth in a material is much longer than its skin depth, then the decay of the magnetic field into the material can be ignored. In this regime, the effect of the penetration of the magnetic field into the material on the heating is small. For copper, a $1 \mu$ s pulse creates a diffusion depth $D=10.8 \mu \mathrm{m}$ as compared to $\delta=0.6 \mu \mathrm{m}$ at $f=11.424 \mathrm{GHz}$.

Letting $\delta \rightarrow 0$, Eq. (4) becomes

$$
\left.\Delta T(z=0, t)\right|_{\delta \rightarrow 0}=\frac{1}{\rho c_{\varepsilon} \sqrt{\pi \alpha_{d}}} \int_{0}^{t} \frac{d t^{\prime}}{\sqrt{t-t^{\prime}}} \frac{d P\left(t^{\prime}\right)}{d A} .
$$

This is the same result if we had considered a prescribed surface heat flux $d P(t) / d A$ with no volume energy generation. For a $1 \mu \mathrm{s}$ pulse, Fig. 10 shows the temperature rise on the surface normalized to the temperature rise at

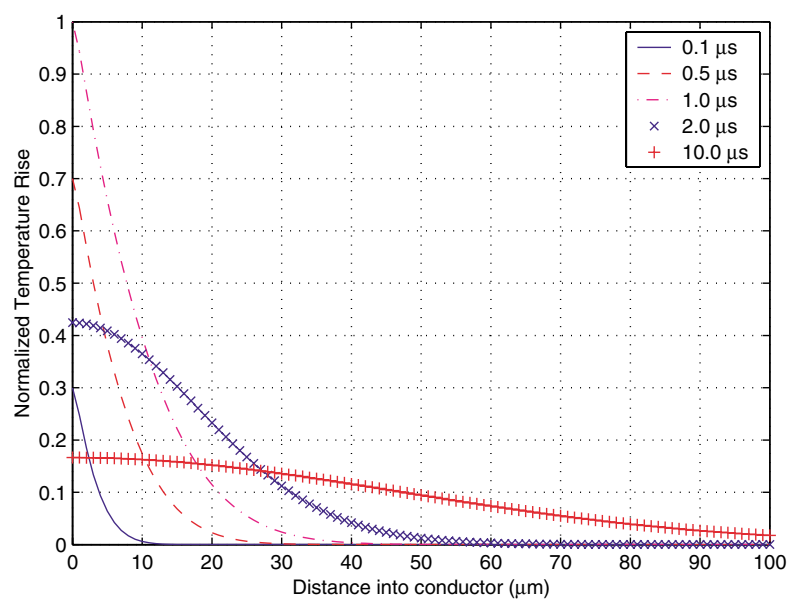

FIG. 10. (Color) The temperature rise at various times in the conductor normalized to the surface temperature at $t=1.0 \mu \mathrm{s}$, for a pulse length of $t_{p}=1.0 \mu \mathrm{s}$. 

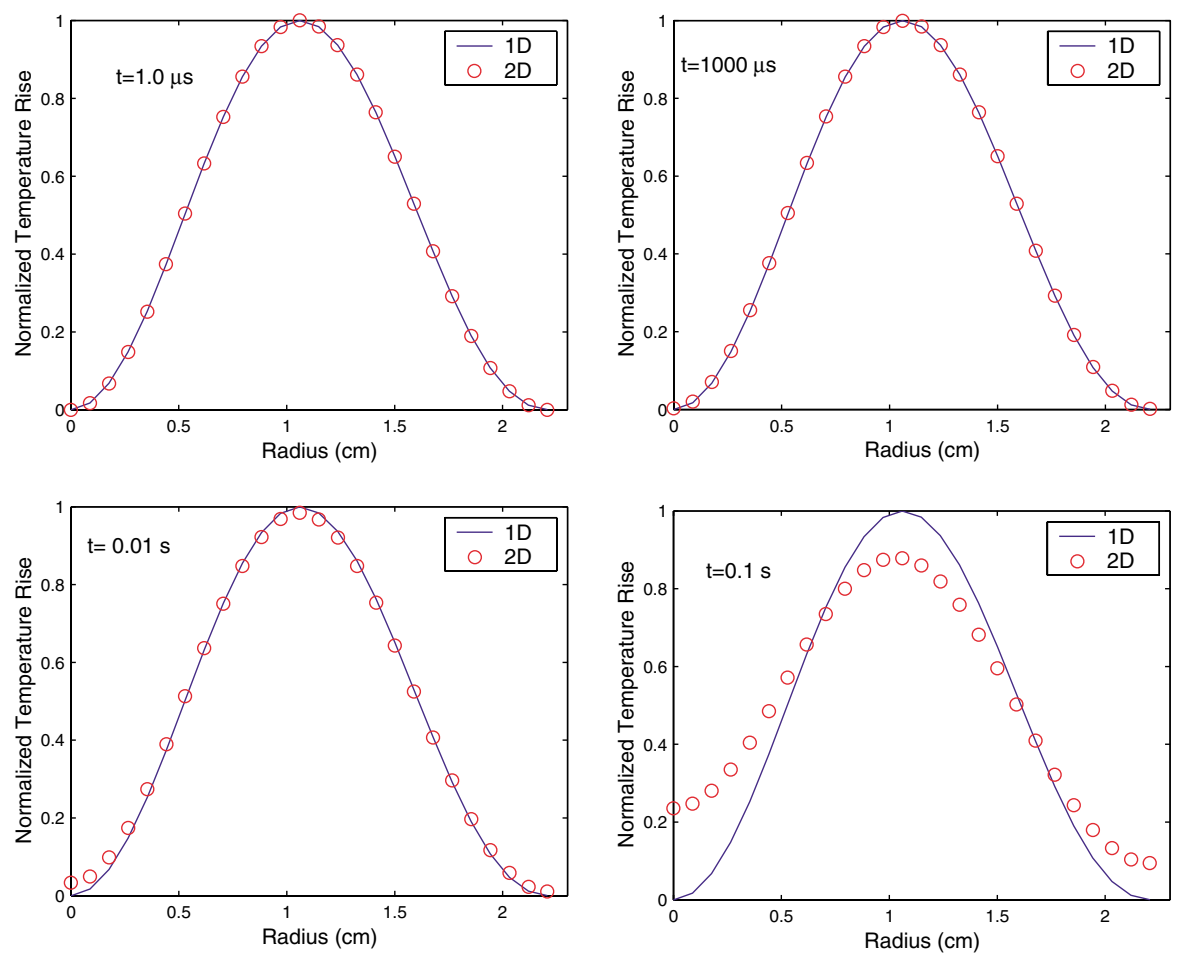

FIG. 11. (Color) 1D and 2D temperature rises of the cavity end caps for various pulse lengths normalized to the maximum temperature rise for $1 \mathrm{D}$.

$1 \mu \mathrm{s}$. The error from ignoring the skin depth on the temperature rise is less than $3 \%$.

Equation (4) assumes the surface is semi-infinite; however, the cavity surface is two dimensional. When a full analysis is done [17], the error in using Eq. (4) to predict the temperature rise on every part of the cavity surface is small for short times. Figure 11 depicts this comparison for the cavity end caps. For times of a $1000 \mu \mathrm{s}$ or less, the one-dimensional approximation is good.

\section{B. Pulsed heating of test cavity}

The calculations shown previously assumed that all the thermal parameters were constant. For temperature rises of $300 \mathrm{~K}$, the specific heat increases by about $5 \%$ and the thermal conductivity decreases by about $5 \%$. The error for not including this temperature dependence in the calculation becomes $1 \%$ [17]. Hence, Eq. (4) is valid by taking $\alpha_{d}$ out of the integral.

The electrical resistivity is an important parameter for the surface temperature rise. Its (linear) dependence on temperature causes the unloaded $Q$ of the cavity to decrease over the length of a pulse. Since a temperature rise of $100 \mathrm{~K}$ will cause the resistivity to increase by $40 \%$, the temperature dependence must be taken into account when calculating the pulsed temperature rise. Its influence can be seen from the local power dissipation

$$
\frac{d P(t)}{d A}=\frac{1}{2} R_{s}|H(t)|^{2}
$$

where $R_{s}=\sqrt{\pi \mu f \rho_{\text {res }}}$.

The calculation is also complicated by the magnetic field's dependence on $Q$. The cavity behaves as a damped, driven oscillator, so the amplitudes of the fields are affected by the fill time. The details of this calculation are fully explained in [17]. Using the cavity parameters given in Table II, Fig. 12 shows the effect of including the temperature dependence of $\rho_{\text {res }}$ for a $10 \mathrm{MW}, 1 \mu$ s pulse on the cavity end caps. The inclusion of this effect increased the temperature rise by $10 \%$.

Notice after a time longer than three pulse lengths, the temperature rise on the surface decays to within $30 \%$ of its maximum value. Since there is approximately $17 \mathrm{~ms}$ between pulses, the temperature rise decays close to zero by this time. There will be some average temperature rise due to the combined effect of many pulses; however, water cooling stabilizes this temperature rise to about $10 \mathrm{~K}$ above room temperature [17].

The degradation in the unloaded $Q$ for each mode is calculated from [17]

$$
\frac{1}{Q_{0}(T)}=\frac{1}{\sqrt{4 \pi \mu f}} \int_{S_{w}} d S \sqrt{\rho_{\text {res }}[T(\vec{r})]}|\vec{H}(\vec{r})|^{2},
$$

where $S_{w}$ is the entire cavity surface and $\vec{r}$ is a point on 


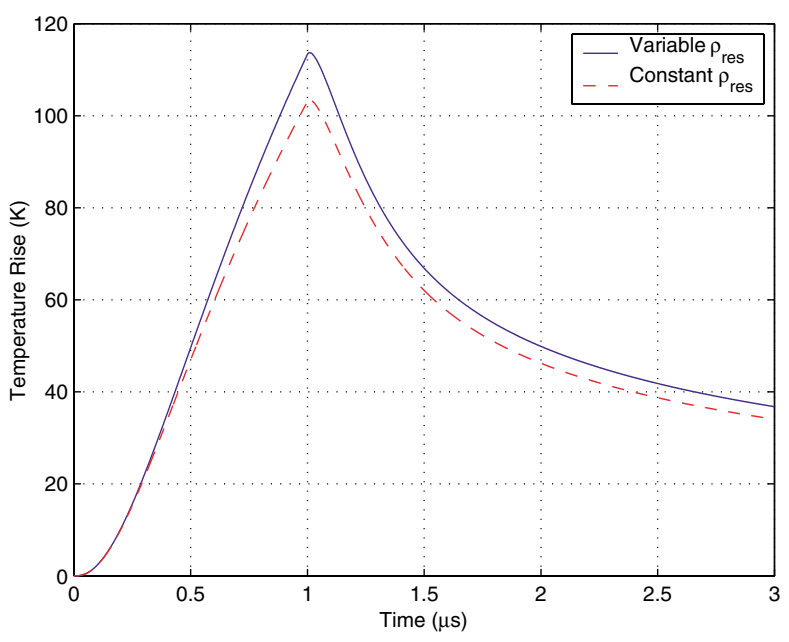

FIG. 12. (Color) Comparison of variable $\rho_{\text {res }}$ with constant $\rho_{\text {res }}$ on the temperature rise of the cavity end caps for a $10 \mathrm{MW}, 1 \mu \mathrm{s}$ pulse.

the surface. For the above example, the $Q$ change for both modes is shown in Fig. 13.

The amplitude and phase of the reflected power from the $\mathrm{TE}_{012}$ mode will change as its unloaded $Q$ degrades over the time of a high-power pulse. The amplitude change is due to a change in the coupling to the mode. The phase shift is due to the change in the resonance frequency which depends on two effects. The first and major effect is the dependence of the cavity's resonant frequency on $Q$. This effect can be intuitively grasped by realizing that the unloaded $Q$ depends on geometry. A finite skin depth means the effective volume that contains electromagnetic fields is bigger than the volume in vacuum. As the $Q$ changes, so will the skin depth and this effective volume. Hence the frequency changes accord-

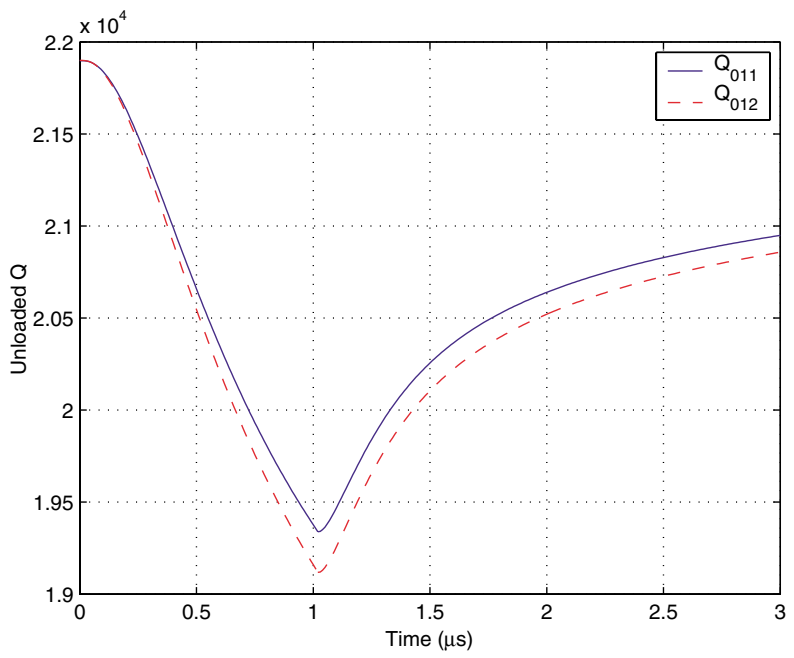

FIG. 13. (Color) Change in $Q_{0}$ for both $\mathrm{TE}_{011}$ and $\mathrm{TE}_{012}$ modes for a $10 \mathrm{MW}, 1 \mu \mathrm{s}$ pulse. ingly. The second effect is due to any deformation that might occur after the pulse turns off.

A calculation of the expected signal looking through the diagnostic port from the $\mathrm{TE}_{012}$ mode is shown in Fig. 14 for a $10 \mathrm{MW}, 1 \mu \mathrm{s}$ pulse. In this case, we have assumed coupling through the diagnostic port of $\beta=1$, and, in addition, leakage out the high-power port with coupling $\beta=0.22$. It is to be noticed that the extrema of these curves occur some time after the pulse shuts off. This lag time is on the order of the cavity fill time.

The change in the unloaded $Q$ and resonant frequency of the $\mathrm{TE}_{012}$ mode is extracted from measurements of the amplitude and phase of the reflected power [17]. The maximum temperature rise on the end caps can be extracted from Eq. (7) with two assumptions. The first assumption is knowledge of the field pattern of the mode. This is well known on the end caps and the majority of the cylindrical sidewall. The only place where it is not known analytically is near the coupling apertures. The apertures modify the fields locally. The second assumption is the ratio between the maximum temperature rise on the cavity end caps to the maximum temperature rise
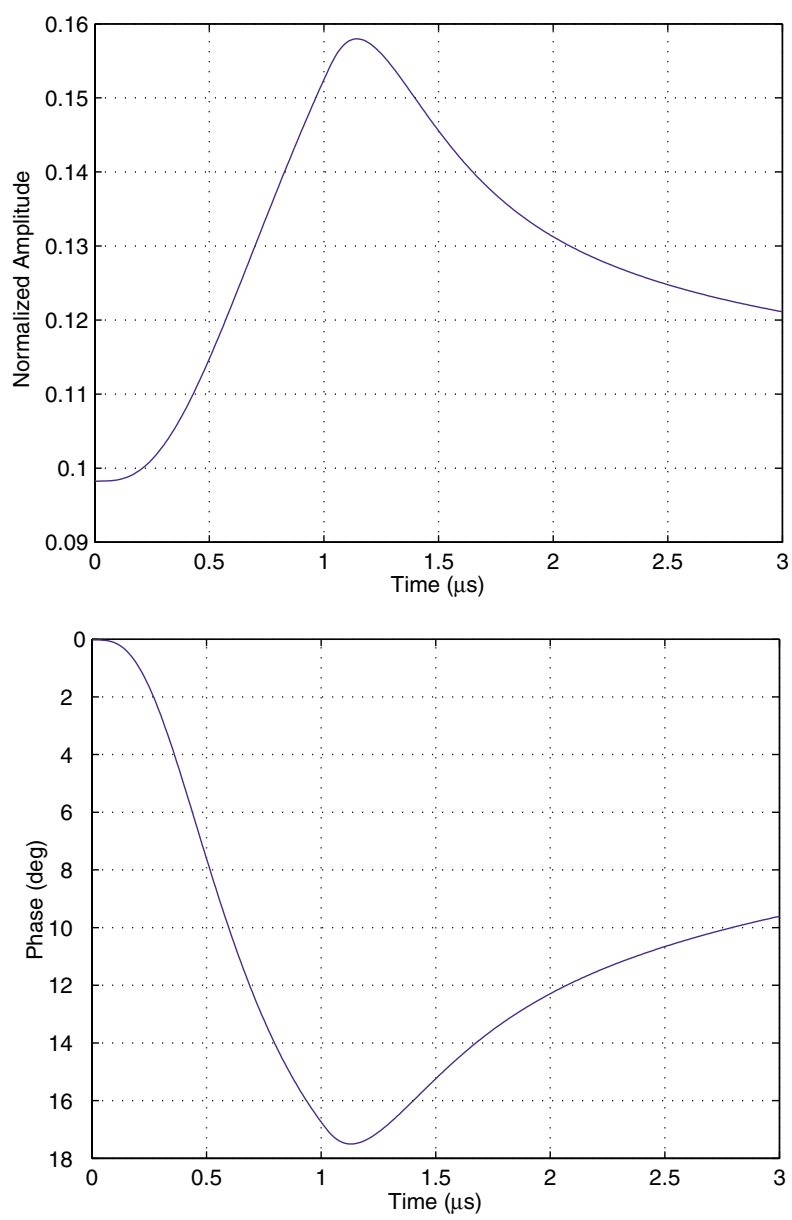

FIG. 14. (Color) Magnitude and phase of the reflected signal with respect to the input signal due to the change in $Q_{0}$ of the $\mathrm{TE}_{012}$ mode. 
on the sidewall which is approximately 2 for the dimensions used. Since changes in unloaded $Q$ of the $\mathrm{TE}_{012}$ mode is 8 times more sensitive to resistivity changes on the end caps as compared to the sidewall, the overall error due to this assumption is less than $1 \%$. See [17] for more details.

\section{EXPERIMENTAL RESULTS}

Results are presented from two different high-power tests. The first test used the two-cavity setup depicted in Fig. 8 which provided four end caps to analyze afterwards. The second test used the one-cavity setup which provided only two end caps but made the experiment easier to run.

\section{A. First test}

In this particular run, we used an XL-3 50 MW klystron that was limited to $1.25 \mu$ s pulses at a repetition rate of $60 \mathrm{~Hz}$. The vacuum in the cavities was approximately $10^{-9}$ Torr. Because of interference of other modes with the $\mathrm{TE}_{012}$ mode, the diagnostic to measure the pulsed temperature rise was not used in this run.

\section{Run-up procedure}

The entire experimental run occurred over a twomonth period in which it took approximately two weeks to reach 8.5 MW of input power to each cavity due to outgassing from the copper surface and rf breakdown. We did not go above $8.5 \mathrm{MW}$ due to the extensive rf breakdown at the high-power coupling aperture.

During the experiment, the cavities were tuned to each other in a way that their resonant frequencies would match after thermal expansion due to average heating. At this input level, the temperature rise due to average heating on the end cap is calculated to be $10 \mathrm{~K}$ and causes a $4 \mathrm{MHz}$ decrease in resonant frequency.

\section{2. rf high-power results}

With the forward and reflected power measurements at $X$ band, the coupling coefficient $\beta$ and the loaded $Q, Q_{L}$, can be determined [17]. The input power to each cavity was measured to be $8.5 \pm 0.6 \mathrm{MW}$. This corresponds to a calculated temperature rise of $120 \pm 10 \mathrm{~K}$. The uncertainties are due to systematic errors in the calibration of the directional couplers and random errors caused by the fluctuation of the crystal detector voltage from noise. The experiment was run at this temperature rise for $5.6 \times 10^{7}$ pulses.

An HP8510C vector network analyzer was used to determine the $\mathrm{rf}$ parameters of each cavity before and after the high-power test. The results, given in Table VI, show a $20 \%$ to $30 \%$ degradation in unloaded $Q$. The damage that resulted on the surface will be shown later. The degradation in external $Q$ is from the increase of the size of the coupling aperture due to damage from pulsed heating. This will also be shown later.

The coupling coefficient, $\beta=Q_{0} / Q_{\mathrm{ext}}$, is determined by measuring the forward and reflected power to and from the cavity. The reflected power is measured near its steady-state value before the rf pulse shuts off. This measurement over the course of the experimental run is shown in Fig. 15 for both cavities. The time-domain measurement of $\beta$ at the end of the run is consistent with the frequency-domain measurement. A few general points can be made about the changing trend in the value of $\beta$ for both cavities. In the first $20 \times 10^{6}$ pulses, $\beta$ increases for both cavities. This must be due to a degradation in $Q_{\text {ext }}$ because $Q_{0}$ can only decrease due to damage. After $20 \times 10^{6}$ pulses, $\beta$ decreases for cavity 1 and increases more slowly for cavity 2 . This demonstrates that the degradation of $Q_{0}$ from surface damage has become dominant. The overall decreasing trend for cavity 1 as compared to the increasing trend demonstrated for cavity 2 is supported by the data given in Table VI. Cavity 1 suffered a smaller change in $Q_{\text {ext }}$ than cavity 2 but suffered a greater change in $Q_{0}$. The variation shown in $\beta$ for cavity 2 after $50 \times 10^{6}$ pulses is due to systematic errors.

In principle, $Q_{L}$ can be measured through the exponential decay of the reflected power when the rf pulse turns off and $Q_{0}$ can be determined by combining $\beta$ and $Q_{L}$ measurements. However, that was not possible for a combination of reasons. Pulsed heating affects rf properties and measurements, so the input power had to be lowered to below $2 \mathrm{MW}$ before measurements could be made. The cavities, which were tuned to each other under

TABLE VI. Cavity $Q$ measurements of cavities 1 and 2 using a HP8510C network analyzer before and after the first high-power test. The change in $Q_{\text {ext }}$ is due to the increase in size of the high-power coupling apertures.

\begin{tabular}{ccccc}
\hline \hline Cavity & Initial $Q_{0}$ & Final $Q_{0}$ & Initial $Q_{L}$ & Final $Q_{L}$ \\
\hline 1 & $20350 \pm 520$ & $14360 \pm 300$ & $7670 \pm 110$ & $5010 \pm 50$ \\
2 & $20610 \pm 530$ & $16810 \pm 260$ & $7670 \pm 110$ & $5010 \pm 50$ \\
Cavity & Initial $\beta$ & Final $\beta$ & Initial $Q_{\text {ext }}$ & Final $Q_{\text {ext }}$ \\
1 & $1.65 \pm 0.06$ & $1.87 \pm 0.05$ & $12320 \pm 520$ & $7690 \pm 260$ \\
2 & $1.69 \pm 0.06$ & $2.36 \pm 0.07$ & $12220 \pm 530$ & $7140 \pm 250$ \\
\hline \hline
\end{tabular}



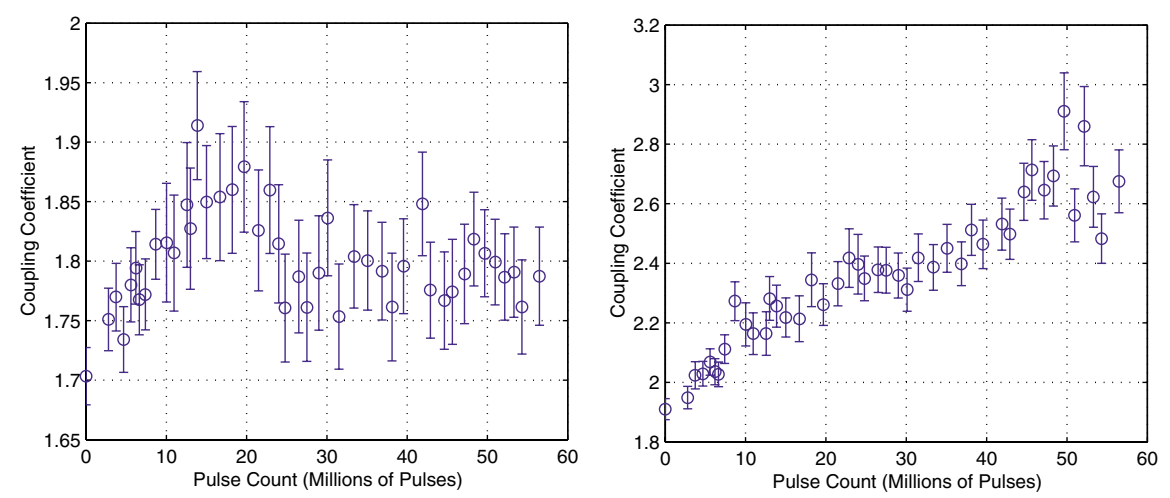

FIG. 15. (Color) Variation of $\beta$ for cavity 1 (left panel) and cavity 2 (right panel) as a function of pulse count at a $120 \mathrm{~K}$ temperature rise on the end cap surfaces. The errors shown are due to random errors only.

full power, become mismatched to each other and, in addition, mismatched to the klystron output cavity. This problem became worse over time as $\beta$ for cavity 2 increased. These mismatches mixed together the characteristic decays, and the variations of $Q_{L}$ and $Q_{0}$ during the experimental run could not be determined [17]. However, the trend in Fig. 15 with the data in Table VI shows a clear degradation of $Q_{0}$ for each cavity.

\section{Visual inspection of copper surface}

At the end of the experimental run, the end caps were removed from the cavities and inspected. One such end cap is shown in Fig. 16. Each end cap is made from polycrystalline OFE copper; therefore, the structure on the surface is made from crystal grains whose lattices are oriented in a generally random direction with respect to each other. Figure 16 shows an obvious visual change in the crystal grains in the region where the temperature rise is maximum, which is approximately halfway between the center and outer radius of the end cap. In this area in which there is high-amplitude cyclic straining, steps may form in crystal grain boundaries with height differences on the order of microns [18]. This would change the direction of light specularly reflected from the surface, which will cause a contrast between the grains in the fatigued area as compared to the rest of the surface.

Also visible on the surface are black patches that appear on the half of the end cap that is closest to the highpower coupling aperture. These patches are most likely due to multipacting as they appear to occur in pairs. Electrons would orbit around the radial magnetic field lines along the end cap. According to GDFIDL simulations, the maximum magnetic field on the end cap at the azimuth of the high-power coupling aperture is 5\% higher than the point diametrically opposite of it. Since the
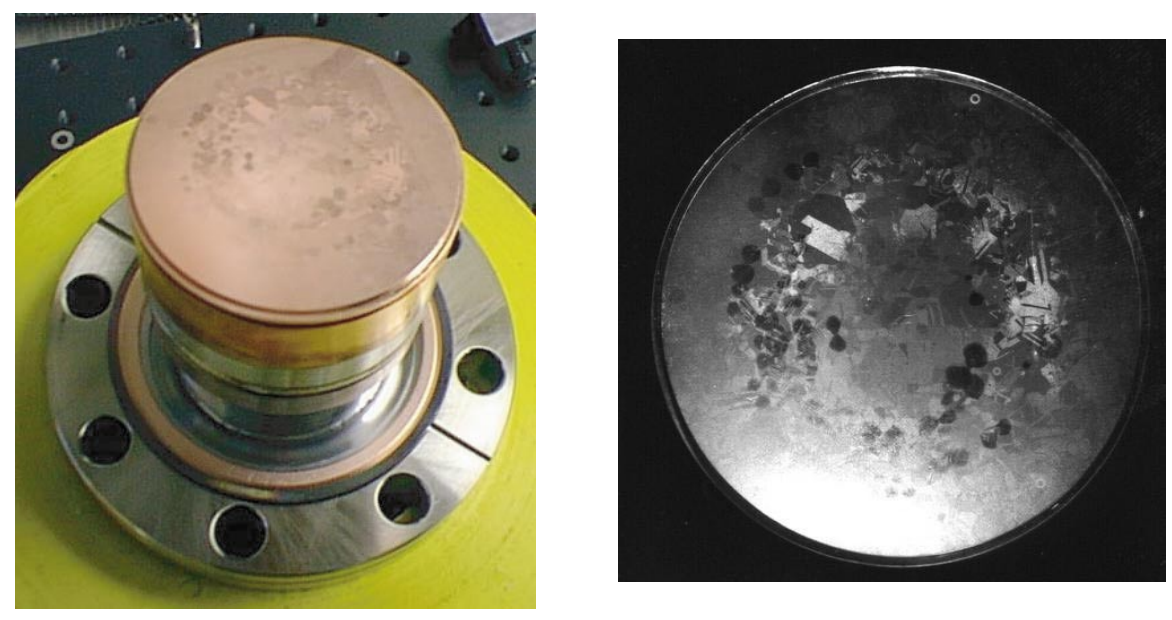

FIG. 16. (Color) Picture of one end cap from the first high-power test. A change in the crystal grains is visible even in the region where the temperature rise was lower by $10 \%$. The region of the end cap nearest the high-power coupler is on the lower left in the right-hand picture. Tarnish from the sputtering of copper is on the lower part of the end cap. 
energy of multipacting electrons is restricted, this could explain why the patches occur only on half of the end cap surface nearest the high-power coupling aperture.

There is also a tarnish visible on the surface. The tarnish is thicker at the outer radius and at the azimuth closest to the high-power coupling aperture. As will be shown in the next section, scanning electron microscope studies show this tarnish to be copper globules that were sputtered onto the surface. Because these globules may add to the surface roughness of the cavity, they may contribute to the degradation in $Q_{0}$ shown in Table VI.

\section{Internal cross section}

Cracks nucleated at the surface may propagate into the interior of the metal. One end cap was cut internally along a diameter and polished to view its cross section. Cracks were only found near the area of maximum temperature rise. Less than ten cracks were visible at a microscope magnification of 1000 and only two cracks are longer than $10 \mu \mathrm{m}$. These cracks are shown in Fig. 17. These cracks were first created at the surface and then propagated internally, because higher stresses and strains occur at the surface than in the interior of the metal [19]. Only a few cracks will propagate internally as compared to the amount of cracks that are nucleated at the surface.

Slip will occur when the shear stress along a slip plane is above a critical value [11]. Since the normal stress axis occurs parallel to the end cap surface, the maximum shear stress occurs along a plane inclined at $45^{\circ}$ to the surface. Since the end cap is made from polycrystalline copper, only grains that have slip planes oriented close to this angle will show slip if the stress is large enough. The cracks in Fig. 17 begin propagating at roughly $45^{\circ}$ to the surface within the first few microns into the surface, which is known as stage I crack propagation. Also seen to occur is stage II crack propagation in which cracks propagate perpendicular to the surface and perpendicular to the stress axis [18].

\section{Scanning electron microscope inspection of copper end cap surface}

Each end cap was inspected with a scanning electron microscope using secondary scattering with a $5 \mathrm{kV}$ beam. First, scans were taken along a random diameter of each end cap. Cracks are visible only in the region where the maximum heating occurred at a radius of $10.6 \mathrm{~mm}$. The thickness of this region is approximately 4 to $5 \mathrm{~mm}$ for each end cap. No other damage is visible on the surface.

Second, scans were taken around the region of maximum heating at different magnifications. One such region is shown on the left of Fig. 18. Numerous cracks are visible on the surface. The bumps that occur on the surface are due to the copper globules that sputtered onto the end caps from the high-power coupling aperture. For comparison, the center of the end cap is shown on the right of Fig. 18. No cracks are visible in the center where the temperature rise is close to zero. The copper globules sputtered on the surface are most dense near the outer radius where no cracks are visible. This suggests that pulsed heating and not the copper globules is responsible for the nucleation of cracks on the surface.

As mentioned earlier, some crystal grains will show signs of slip if they have the proper orientation. Figure 19 shows an example of these slip bands as striations on the surface. Slip bands are created from stress relaxation of the surface due to plastic deformation, which results in
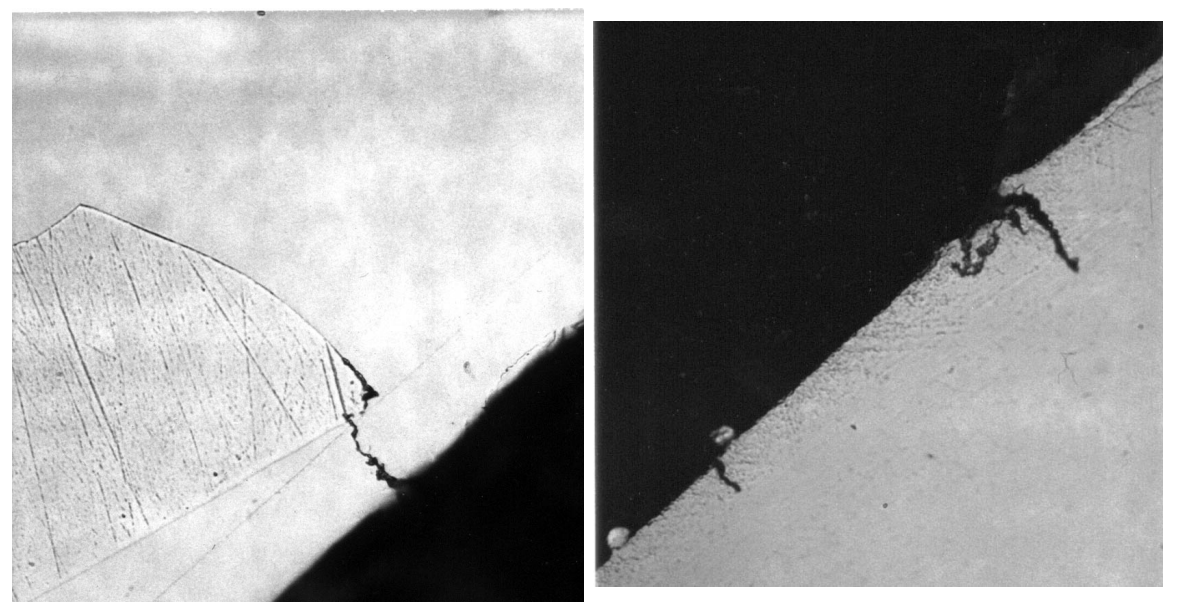

FIG. 17. An internal cross section of one end cap in the region of maximum temperature rise on the surface. The end cap surface is the boundary between the white and black regions of the pictures. The crack on the left propagated $25 \mu \mathrm{m}$ into the surface before reaching an internal grain boundary. The crack on the right propagated $15 \mu \mathrm{m}$ into the surface. 

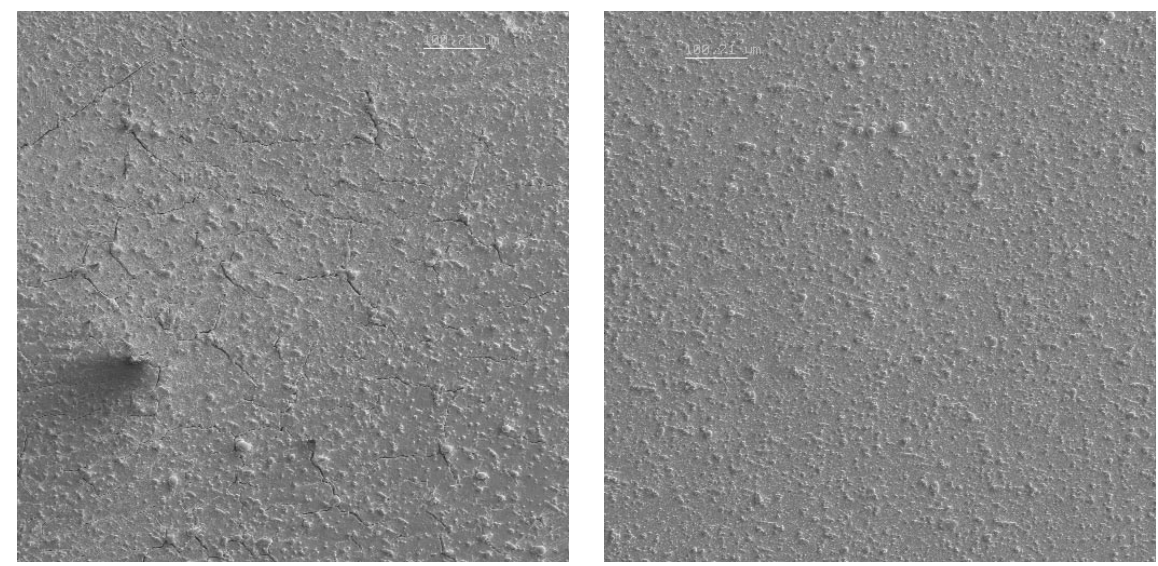

FIG. 18. Comparison snapshot of a region of maximum temperature rise on the left and a region of zero temperature rise on the right for one end cap. The length scale is $100.7 \mu \mathrm{m}$.

surface intrusions and extrusions. Surface intrusions create a notch effect for possible nucleation of microcracks as demonstrated on the right of Fig. 19 [18]. In general, crack propagation along the surface is perpendicular to fatigue striations [19].

Grain boundaries are another common site for crack nucleations [19]. Figure 20 is an example of cracks along grain boundaries. In the picture on the left, the transition between the two different textures on the surface is shown by the red arrows. The texture on the right-hand side of the picture is due to slip bands. A close-up of the grain-boundary crack is in the right-hand picture. Notice that the striations of the slip bands end at the crack. Grain boundaries act as natural barriers for slip bands, because the neighboring grains usually have incompatible orientations that prevent slip bands propagating across the boundary. The enhanced stress from this incompatibility may lead to crack nucleation at the grain boundary. See [17] for more pictures of cracks and slip bands.

Scans were completed along random diameters of each end cap at the same magnification of 100. Each snapshot corresponded to an area approximately 0.9 by $0.9 \mathrm{~mm}$. Figure 21 shows the results of these scans.

In general, the scans indicate that surface roughening from fatigue is a precursor for crack nucleation, because most of the regions containing cracks are within the regions of surface roughness. Also, the bands generally surround the expected area of maximum temperature rise at the radius of $10.6 \mathrm{~mm}$. The widths of these regions are listed in Table VII. The variation in the widths of damage reported in Table VII among each end cap is due to the location of the scanned diameter with respect to the azimuth of the high-power coupling aperture. The widths are generally larger when the scanned diameter is closer to the aperture. This feature is largely explained by the
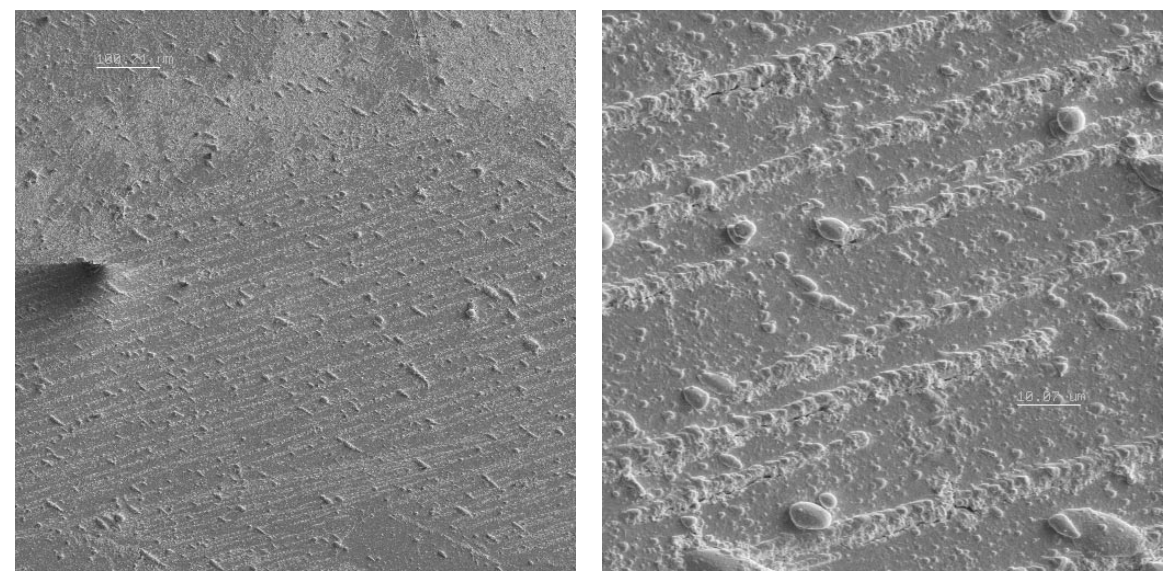

FIG. 19. Fatigue slip bands on a particular crystal grain in the area of maximum temperature rise. The picture on the right is a close-up of these slip bands in which some microcracks are evident at the base of the slip bands. The scale is $100.7 \mu \mathrm{m}$ on the left and $10.1 \mu \mathrm{m}$ on the right. 

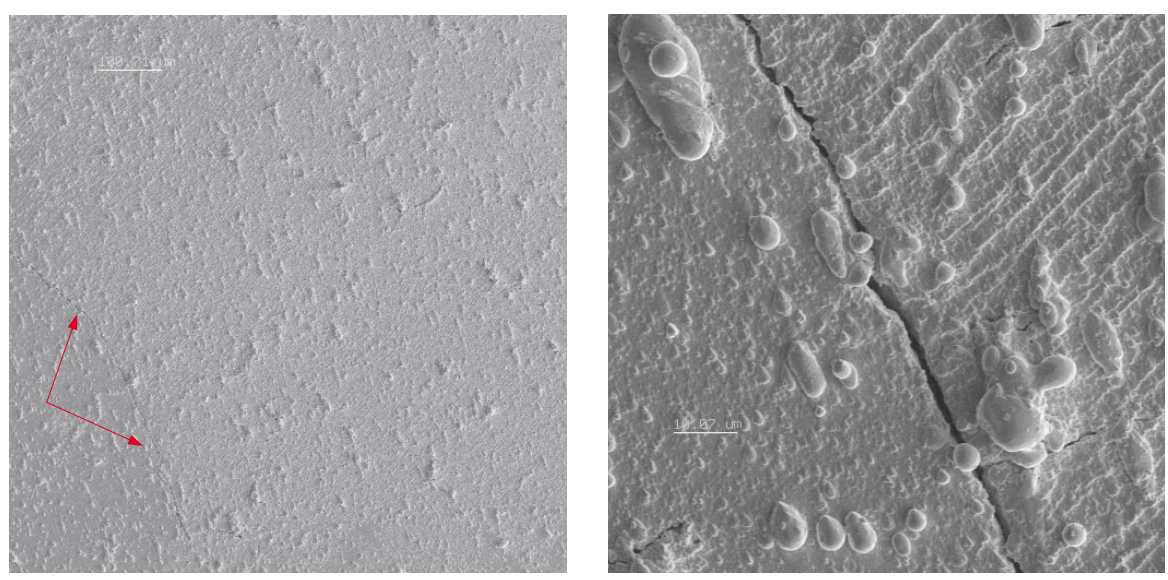

FIG. 20. (Color) Grain-boundary cracks in the area of maximum temperature rise. The grain boundary is the transition between the two different textures on the surface shown by the red arrows. The texture on the right-hand side of the picture on the left is due to slip bands. A close-up of one crack is in the right-hand picture where the grain boundary is clear. The length scales are $100.7 \mu \mathrm{m}$ and $10.1 \mu \mathrm{m}$, respectively.
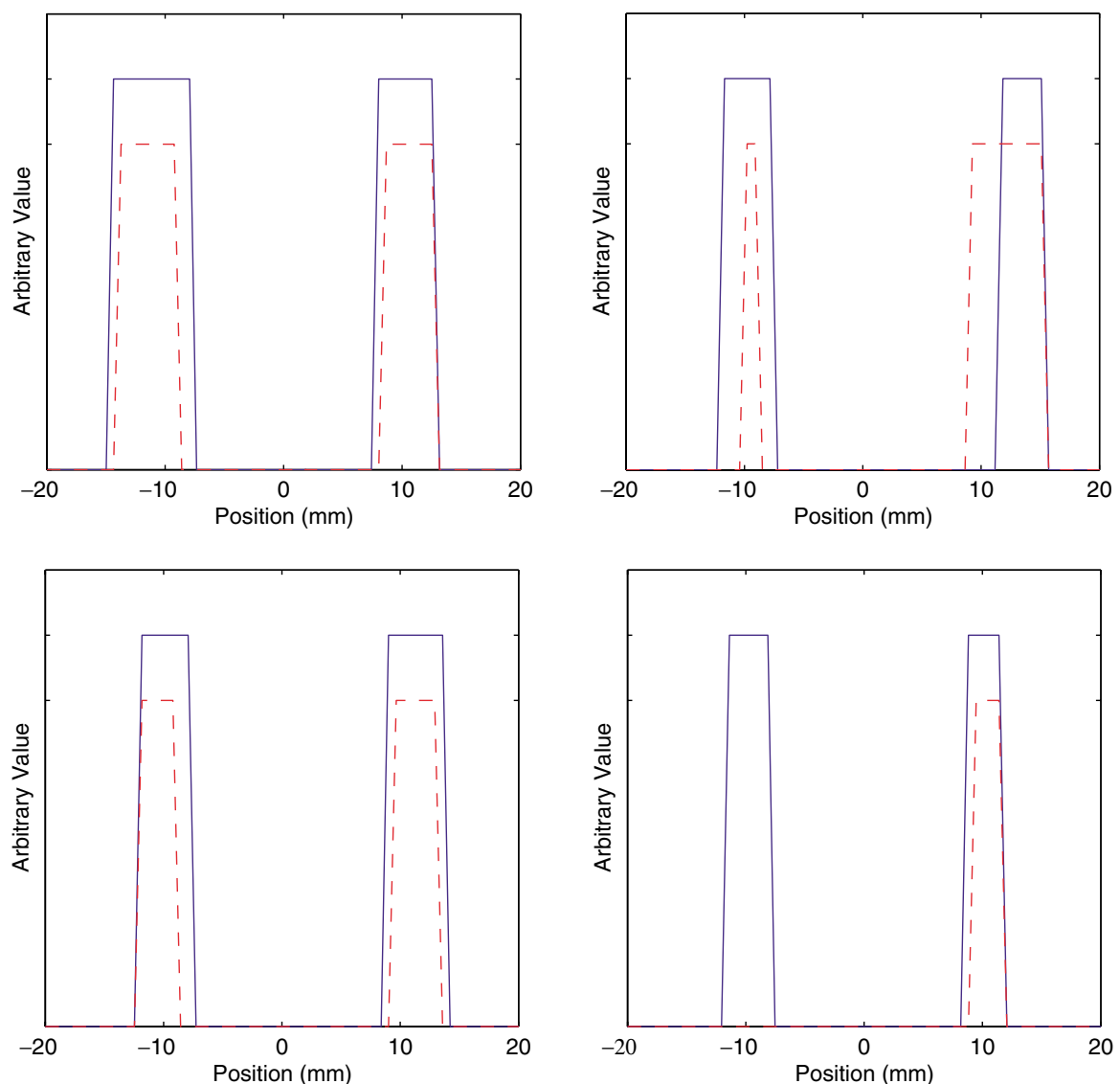

FIG. 21. (Color) Random scans along diameters of each end cap. The solid lines correspond to regions of surface roughening and the dotted lines are for regions that contain cracks on the surface. The centers of the end caps are at position $0 \mathrm{~mm}$.

higher temperature rise in this area due to the higher surface magnetic field as explained in Sec. IV A 3.

Numerous pictures were taken in areas that showed many cracks. Table VIII shows the average radius and standard deviation of the positions at which the highest density cracks were found. The maximum temperature rise is expected to occur at approximately $10.6 \mathrm{~mm}$. 
TABLE VII. Widths of regions of surface roughening and cracks for each end cap from the first high-power test.

\begin{tabular}{lcccccccc}
\hline \hline Region & \multicolumn{1}{c}{1} & \multicolumn{2}{c}{2} & \multicolumn{2}{c}{3} & \multicolumn{2}{c}{$\begin{array}{c}4 \\
(\mathrm{~mm})\end{array}$} \\
\hline Roughness & 6.4 & 4.5 & 3.8 & 3.2 & 3.9 & 4.6 & 3.2 & 2.6 \\
Cracks & 5.1 & 3.8 & 0.7 & 5.8 & 2.6 & 3.2 & 0 & 1.9 \\
\hline \hline
\end{tabular}

TABLE VIII. Average radius and standard deviation of crack positions for each end cap.

\begin{tabular}{ccc}
\hline \hline End cap & Average radius $(\mathrm{mm})$ & Standard deviation $(\mathrm{mm})$ \\
\hline 1 & 10.4 & 1.3 \\
2 & 11.3 & 0.9 \\
3 & 10.5 & 1.4 \\
4 & 11.1 & 1.1 \\
\hline \hline
\end{tabular}

\section{Damage to high-power coupling aperture}

There was damage to the high-power coupling apertures that provided unexpected information about temperature limitations from pulsed heating. Figure 22 shows that significant damage has occurred to the highpower coupling aperture of cavity 1 . Simulations using the computer code HFSS [20] show that the strength of the magnetic field on the aperture will cause a temperature rise of $250 \mathrm{~K}$. The magnetic field wraps around the edge of the aperture and is enhanced. The damage on the aperture occurs at this spot. The electric field is generally azimuthal except near the aperture in which it is curved towards the surface. The electric field at the aperture is approximately $85 \mathrm{MV} / \mathrm{m}$, which is sufficient to cause rf breakdown. Pitting has occurred at this spot.

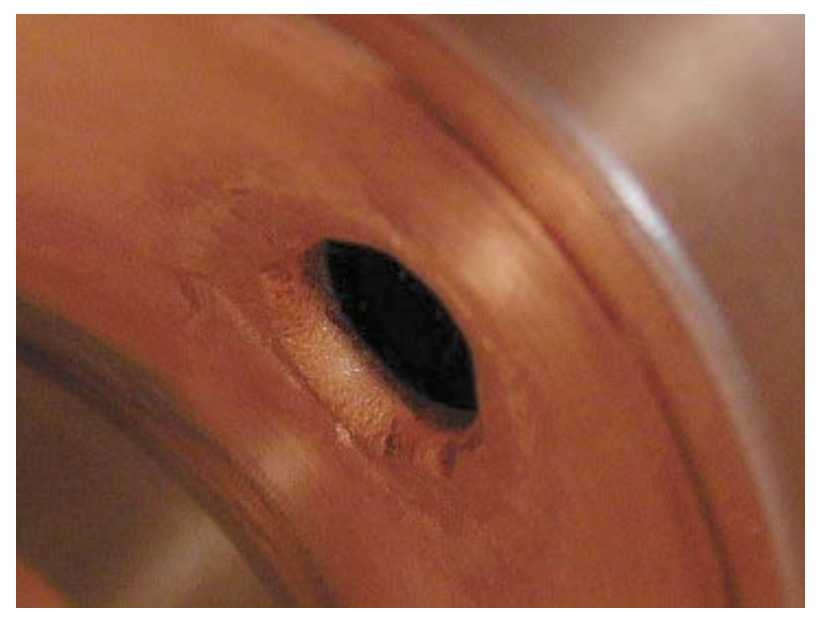

FIG. 22. (Color) A picture of the high-power coupling aperture of cavity 1 after the first high-power test. The aperture has been rounded in the direction of the magnetic field. The electric field has caused pitting to occur on either side of the aperture at right angles to the rounding.
Since the damage on the aperture appears to occur in the direction of the surface magnetic field, a likely explanation for the damage is fatigue due to pulsed heating. As cracks develop in the aperture region, the heating will increase due to the larger electrical resistivity. The increased heating will cause more cracks to develop and increase the size of cracks that already exist. Eventually local melting will result. Metal will evaporate and spray from these cracks [9]. This result indicates that extensive damage will occur at a temperature rise of $250 \mathrm{~K}$ for $50 \times 10^{6}$ pulses. This is an important limit.

\section{B. Second test}

The second high-power test was conducted with cavity 1 from the previous high-power test using new OFE copper end caps after the cavity was cleaned for vacuum. Since most of the cavity was processed with high power previously, it took only one week to reach full power. The vacuum in the cavity throughout the run is $10^{-9}$ Torr.

The same klystron was used but in a different test area. In this area, the diagnostic measurement setup was closer to the modulator which added more noise to the crystal detector voltages. Since the forward power was measured with a peak-power analyzer, this did not affect that measurement. However, it increased the error in the determination of the coupling coefficient and the loaded $Q$ in the time domain.

\section{High-power results}

The cavity was excited with $7.3 \pm 0.3 \mathrm{MW}, 1.5 \mu \mathrm{s}$ pulses at $60 \mathrm{~Hz}$ for $8.6 \times 10^{7}$ pulses. This corresponds to a calculated maximum temperature rise on the end caps of $82 \pm 3 \mathrm{~K}$. Table IX gives the rf parameters of the $\mathrm{TE}_{011}$ mode before and after the test. The table shows a $Q_{0}$ degradation of $5 \%$ and a $Q_{\text {ext }}$ degradation of $10 \%$. The rf parameters for the $\mathrm{TE}_{012}$ mode are given in Table $\mathrm{X}$. For the $\mathrm{TE}_{012}$ mode, port 1 corresponds to the low-power diagnostic port and port 2 corresponds to the high-power port. As Table $\mathrm{X}$ demonstrates, there is some leakage of the $\mathrm{TE}_{012}$ mode out the high-power port due to a nonzero coupling. Further tests indicate that it leaks as a $\mathrm{TE}_{10}$ mode in the waveguide, which means the leakage is caused by asymmetry about the large high-power coupling aperture. This leakage must be taken into account when determining the rf parameters in the time domain.

Since the end caps are cut to a mirror finish and the cavity sidewall has had damage from the previous highpower test, we expect the resistivities on the end caps and the sidewall to be different. Assuming the resistivities are constant along each surface, the measured value of $Q_{0}$ for each mode determines these resistivities. The results are given in Table XI. The theoretical resistivity at room 
TABLE IX. Cavity $Q$ measurements of $\mathrm{TE}_{011}$ mode of cavity 1 using a HP8510C network analyzer before and after the second high-power test. The resonant frequency is $11.425 \mathrm{GHz}$. The errors are due to errors from fits to the data.

\begin{tabular}{|c|c|c|c|c|c|c|c|}
\hline Initial $Q_{0}$ & Final $Q_{0}$ & Initial $Q_{\text {ext }}$ & Final $Q_{\text {ext }}$ & Initial $Q_{L}$ & Final $Q_{L}$ & Initial $\beta$ & Final $\beta$ \\
\hline $17750 \pm 360$ & $16870 \pm 310$ & $6850 \pm 220$ & $6200 \pm 180$ & $4940 \pm 40$ & $4530 \pm 40$ & $2.59 \pm 0.07$ & $2.72 \pm 0.06$ \\
\hline
\end{tabular}

TABLE X. Cavity $Q$ measurements of $\mathrm{TE}_{012}$ mode of cavity 1 using a HP8510C network analyzer before and after the second high-power test. The resonant frequency is $17.850 \mathrm{GHz}$.

\begin{tabular}{cccc}
\hline \hline Initial $Q_{0}$ & Final $Q_{0}$ & Initial $Q_{L}$ & Final $Q_{L}$ \\
\hline $20380 \pm 360$ & $18570 \pm 320$ & $10940 \pm 140$ & $10140 \pm 120$ \\
Initial $Q_{1, \mathrm{ext}}$ & Final $Q_{1, \mathrm{ext}}$ & Initial $Q_{2, \mathrm{ext}}$ & Final $Q_{2, \mathrm{ext}}$ \\
$28180 \pm 850$ & $26920 \pm 910$ & $144500 \pm 5400$ & $130800 \pm 5100$ \\
Initial $\beta_{1}$ & Final $\beta_{1}$ & Initial $\beta_{2}$ & Final $\beta_{2}$ \\
$0.72 \pm 0.02$ & $0.69 \pm 0.02$ & $0.14 \pm 0.005$ & $0.14 \pm 0.005$ \\
\hline \hline
\end{tabular}

TABLE XI. Electrical resistivities of the end caps and cavity sidewall before and after the second high-power test. $\rho_{\text {end }}$ indicates the resistivity along the end cap assuming the entire surface changed. $\rho_{\text {band }}$ indicates the resistivity in a band approximately 7 to $8 \mathrm{~mm}$ in width around the area of maximum temperature rise assuming there is no damage outside the band. The reported errors are due to errors in the determination of $Q_{0}$ for each mode.

\begin{tabular}{cccc}
\hline \hline & $\rho_{\text {end }}$ & $\rho_{\text {band }}$ & \\
Test & $\left(\times 10^{-8} \Omega \mathrm{m}\right)$ & $\left(\times 10^{-8} \Omega \mathrm{m}\right)$ & $\rho_{\text {wall }}$ \\
$\left(\times 10^{-8} \Omega \mathrm{m}\right)$
\end{tabular}

temperature for pure copper is $1.7 \times 10^{-8} \Omega \mathrm{m}$, which is close to the initial value measured for the end caps. If the resistivity is constant along the end cap surface, then the resistivity increased by approximately $25 \%$.

Constant resistivity is a good approximation before the high-power test; however, the damage to the end caps shown later indicates that the resistivity will be different within a band around the area of maximum temperature rise. The visible band of damage has a width of 7-8 $\mathrm{mm}$, corresponding to temperature rises from $65-82 \mathrm{~K}$. The resistivity within this band can be found using the $Q_{0}$ measurements and taking $\rho=1.73 \times 10^{-8} \Omega \mathrm{m}$ outside the band. The result, given in Table XI, is $60 \%$ increase in resistivity. This is one bound to the resistivity change due to pulsed heating, and the constant resistivity approximation gives a lower bound of $25 \%$ change. See [17] for a more detailed table.

Since the temperature rise is proportional to the square root of electrical resistivity, the temperature is expected to increase over time as the copper surface becomes degraded from fatigue damage. With these resistivity increases, the maximum temperature rise on the end caps increased between $10 \%$ to $25 \%$ to between $90 \mathrm{~K}$ and $105 \mathrm{~K}$. Over time, the resistivity is expected to increase due to further damage from an increased temperature rise, which leads to thermal runaway.
The measurements of $Q_{0}$ in the frequency domain for the $\mathrm{TE}_{011}$ mode do indicate a degradation due to an increase in the surface electrical resistivity. The timedomain measurements were harmed by noise and systematics. Some of this error is due to a mismatch of the test cavity with the klystron output cavity as well standing waves that exist in the coupling arm of the $55 \mathrm{~dB}$ directional coupler. See [17] for more details. The degradation in $Q_{\text {ext }}$ is due to damage to the high-power coupling aperture; however, it is not as extensive as compared to the first test.

Since the measurement of the rf parameters of the $\mathrm{TE}_{012}$ mode in the time domain occurs between klystron pulses, they do not suffer from modulator noise. However, there is a large scatter in the data partly due to vibrations of the end caps from the flow of cooling water seen as fluctuations in the steady-state value of the reflected power from the diagnostic port. Despite this scatter, Fig. 23 shows a general decreasing trend in $\beta_{1}$ over time. This is expected from the $Q_{0}$ degradation due to fatigue damage on the surface of the end caps. Although there is some degradation of $Q_{\mathrm{ext}, 1}$ from damage to the diagnostic coupling aperture, there is more degradation of $Q_{0}$ resulting in an overall decrease in $\beta_{1}$.

The measurement of $Q_{L}$ for the $\mathrm{TE}_{012}$ in the time domain was made difficult due to the vibration of the 


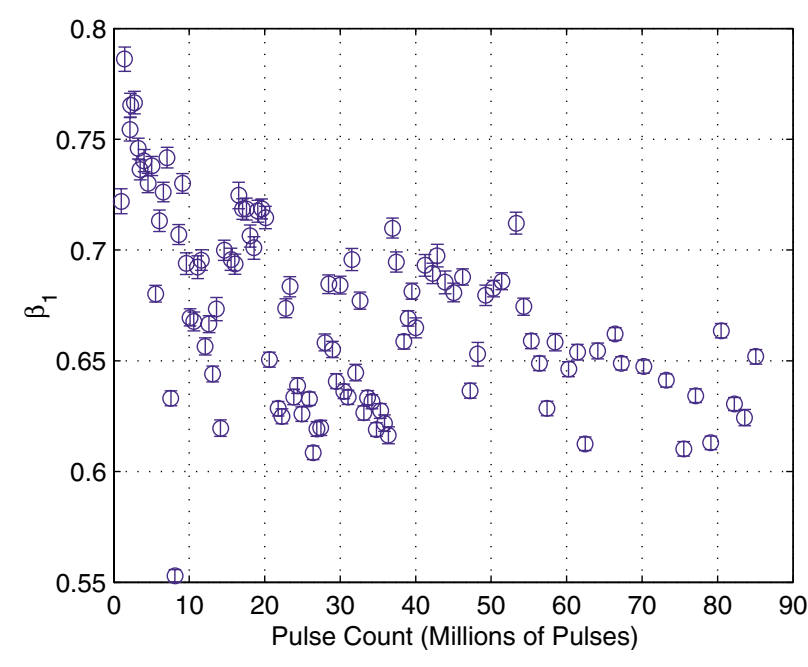

FIG. 23. (Color) Measurement of $\beta_{1}$ during the high-power test for the $\mathrm{TE}_{012}$ mode.

end caps as well as mode leakage out the high-power port. The leakage may have caused reflections from the magic tees and the klystron output cavity that could influence the measurement. Hence, an accurate isolated determination of $Q_{0}$ over time was not possible.

Using the experimental setup described in Sec. II C, the maximum pulsed temperature rise on the surface of the end caps may be inferred from the measurement of the change in $Q_{0}$ of the $\mathrm{TE}_{012}$ mode. The outputs from the quadrature mixer determine the amplitude and phase of the reflected power. From this the change in resonant frequency and $Q$ is determined. From our measurements the change in resonant frequency is mostly due to changes in $Q$ with only small effects due to a change in cavity volume from pulsed thermal expansions. The measurement is made difficult by vibrations of the end caps and modulator noise. The measured signals are low pass filtered with a fast-Fourier transform to reduce the noise. See [17] for details of this measurement.

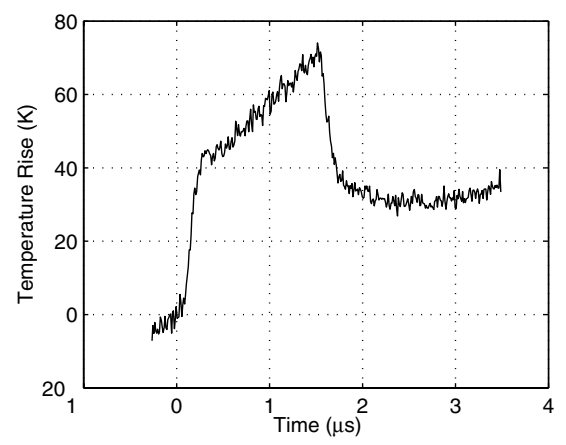

Figure 24 shows the measured maximum temperature rise on the end cap along with the theoretical prediction based on an input power of $7.3 \mathrm{MW}$. The maximum temperature rise is $72 \mathrm{~K}$. This measurement has random and systematic errors. The random errors are associated with residual noise on the waveforms. The systematic errors arise from the error in the resistivity of the end caps reported in Table XI, and the difference between the shapes of the measured and theoretical temperature rises in Fig. 24. Those differences are as follows.

(i) The rapid rise and fall times when the $X$-band power is turned on and off. They are comparable to the cavity fill time.

(ii) The apparent temperature rise after the high-power rf has been turned off.

The actual measurements are changes in amplitude and phase of the reflected $\mathrm{TE}_{012}$ power, and the measured results in Fig. 24 interpret those changes as being caused by temperature rise alone. In fact, other phenomena also contribute to the amplitude and phase changes. The longterm behavior is the initial manifestation of an acoustic effect that is important but does not affect measurement of the maximum temperature rise. Stress waves are launched on the end cap surfaces that cause the end cap positions in the cavity to vibrate. This is seen by a large ramp and a $4 \mathrm{kHz}$ oscillation in the signal that occurs after the rf pulse turns off [17]. The rapid rise and fall of the signal with $X$-band power is not understood. These features were present throughout the data taking indicating that they are not due to changes in surface properties caused by rf pulsed heating. Given the limits of our understanding, the best measurement of the maximum temperature rise is $82 \mathrm{~K}$ based on the input power measurement.

\section{Visual inspection of copper end cap surface}

As with the first high-power test, an obvious visual change in the grains of both end caps is shown in Fig. 25.

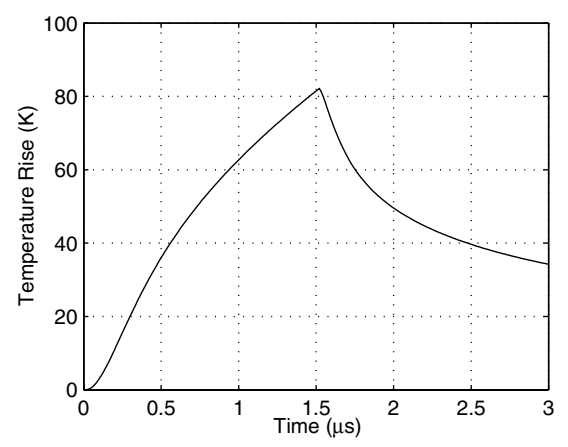

FIG. 24. Measured and theoretical prediction of maximum temperature rise on the end caps during a high-power pulse. Changes in amplitude and phase of the reflected $\mathrm{TE}_{012}$ power are assumed to be caused by temperature rise alone in this measurement. There is discussion of this assumption in the text. The theoretical prediction is based on a 7.3 MW power input. The rf pulse is initiated at time $0 \mu \mathrm{s}$. 

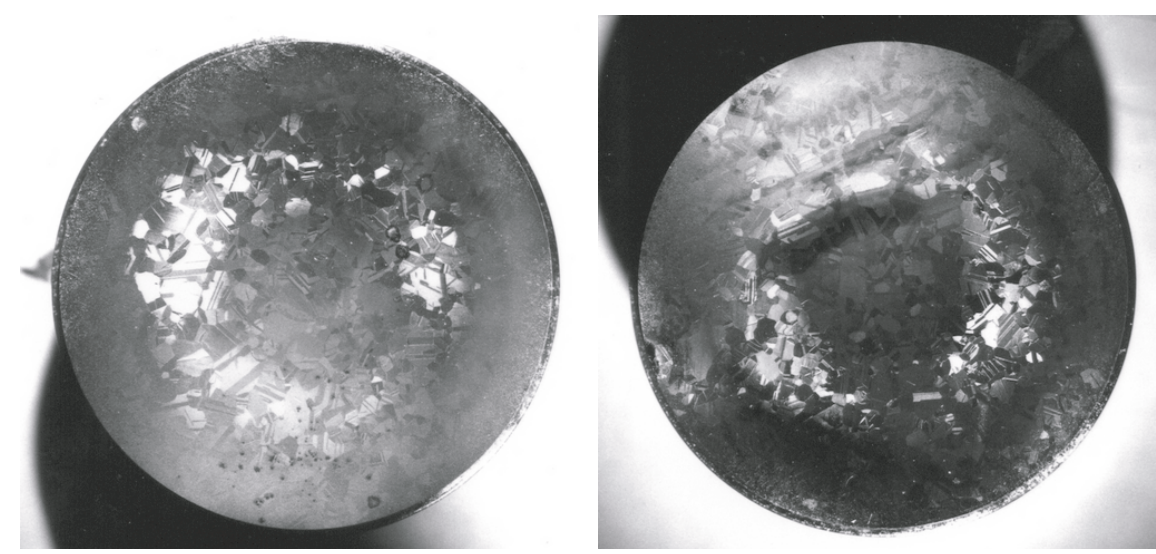

FIG. 25. Surfaces of end caps 1 (left panel) and 2 (right panel) after the second high-power test. The position of the high-power coupling aperture relative to the pictures is the top of end cap 1 and top left of end cap 2.

The width of these regions is between 7 and $8 \mathrm{~mm}$. There is also tarnish on the surfaces as before; however, the amount of copper sputtered from the high-power coupling aperture is much less than the first high-power test because of the lower input power. Therefore, it is reasonable to conclude that most of the resistivity change reported earlier is due to fatigue damage. There is also no visible evidence of multipacting on the surface. This is reasonable, since the surface magnetic field is $15 \%$ less than the first test. In the first test, multipacting occurred only on a half-circle (see Fig. 16) in which a 5\% decrease in the magnetic field is enough to prevent multipacting on the other half-circle.

\section{Scanning electron microscope inspection of copper end cap surface}

As before, the end caps were examined with a scanning electron microscope using secondary scattering with a $5 \mathrm{kV}$ electron beam. The amount of cracks on the surface is smaller than in the first test, which is attributed to a lower pulsed temperature rise.

As a point of comparison, the picture on the left of Fig. 26 shows a region in the area of maximum temperature rise with a multitude of fatigue slip bands including grains with multiple slip. Multiple slips can occur when the crystal axes of the grain are such that the applied shear stress is greater than a critical value on more than one slip system [21]. On the right is a region near the center of the end cap in which the pulsed temperature rise is close to zero. A grain boundary is evident from a change in contrast in the picture; however, no fatigue slip bands or cracks occur in this area.

The results from this experimental run indicate that cracks occur only along grain boundaries. Grain boundaries are clearly seen between areas that contain fatigue slip bands and areas that do not have them. Figure 27 shows such a case in which a crack clearly occurs along a grain boundary in the area of maximum temperature rise. Cracks tend to nucleate at grain boundaries due to
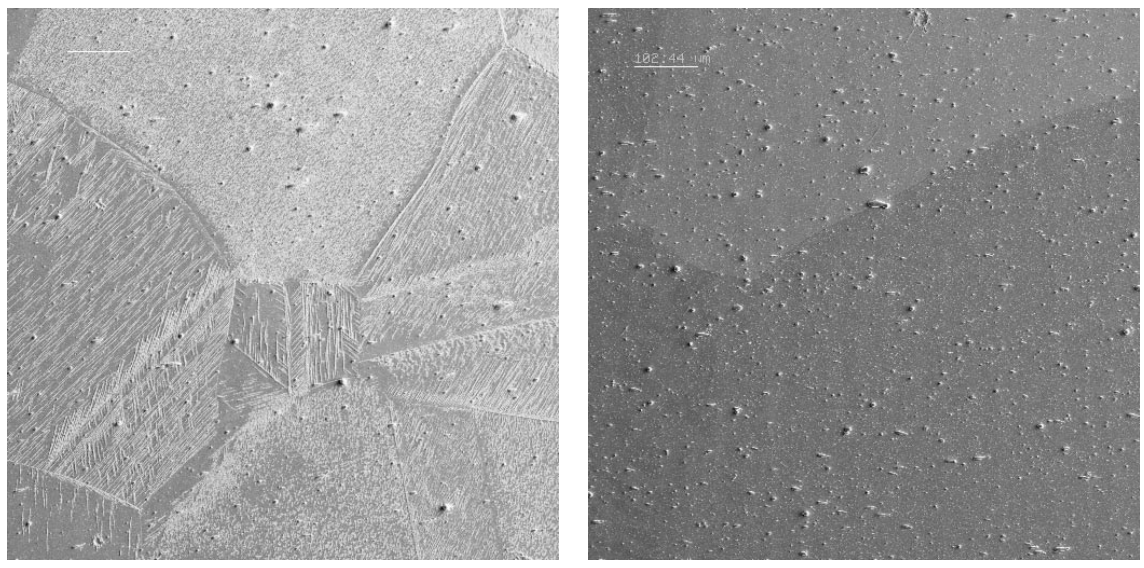

FIG. 26. A comparison between a region in the area of maximum temperature rise (left panel) and the center of the end cap in which the temperature rise is close to zero (right panel). The length scale is $100.7 \mu \mathrm{m}$. 

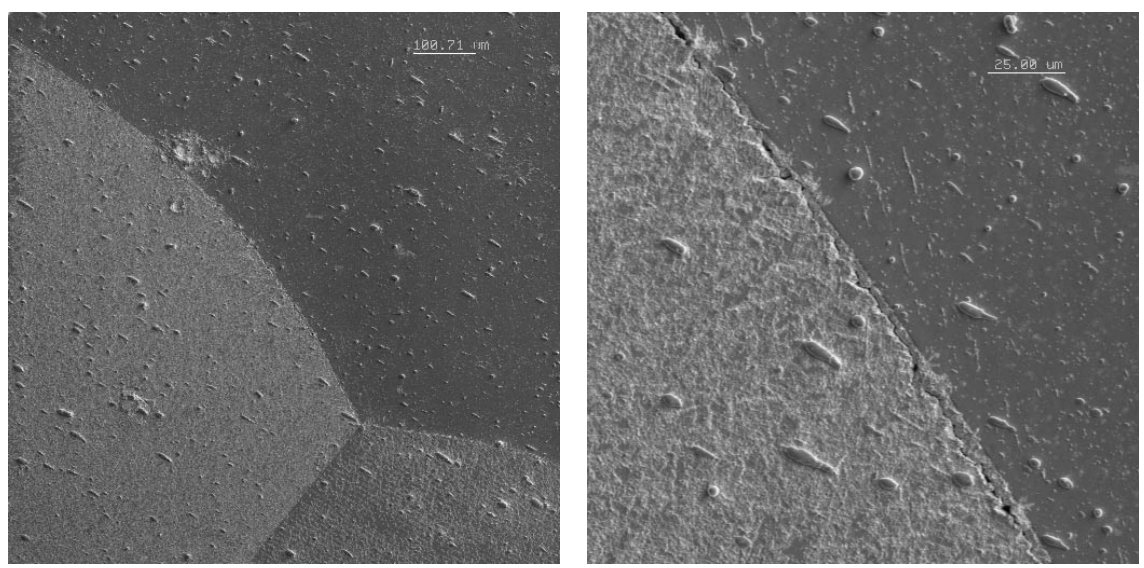

FIG. 27. Grain-boundary cracks in the area of maximum temperature rise. The grain boundary is the transition where the whitening due to slip bands stops. A close-up of one crack is on the right where the grain boundary is clear. The length scales are $100.7 \mu \mathrm{m}$ and $25.0 \mu \mathrm{m}$, respectively.
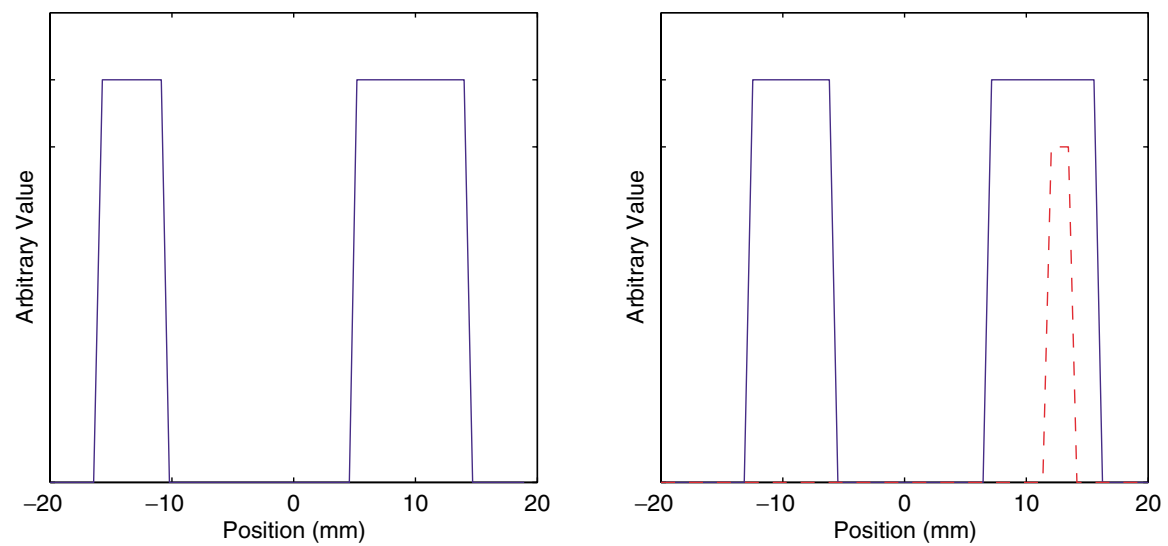

FIG. 28. (Color) Random scans along diameters of each end cap. The solid lines correspond to regions of surface roughening and the dotted lines are for regions that contain cracks on the surface. The centers of the end caps are at position $0 \mathrm{~mm}$.

incompatibility of slip from one grain to the next. Incompatible slip will increase the local stress at a grain boundary. See [17] for more pictures of fatigue slip bands and cracks.

As before, a scan along a random diameter of each end cap at constant magnification of 100 was completed. Each snapshot covered an area approximately 0.9 by $0.9 \mathrm{~mm}$ in size. Arbitrary values were assigned to regions that had cracks or slip bands as with the first test. Figure 28 shows the results of these scans.

TABLE XII. Widths of regions of surface roughening and cracks for end caps 1 and 2 .

\begin{tabular}{lcccc}
\hline \hline Region & $\begin{array}{c}1 \\
(\mathrm{~mm})\end{array}$ & \multicolumn{2}{c}{$\begin{array}{c}2 \\
(\mathrm{~mm})\end{array}$} \\
\hline Roughness & 4.8 & 8.8 & 6.3 & 8.4 \\
Cracks & 0.0 & 0.0 & 0.0 & 1.4 \\
\hline \hline
\end{tabular}

The plots show that surface roughening and slip bands occur around the area of maximum temperature rise at the radius $10.6 \mathrm{~mm}$. The offset that occurs in the plot for end cap 1 is due to an offset of $2 \mathrm{~mm}$ of the scanned diameter from the center of the end cap. The center of the end cap was approximated at the time the diameters were scanned and determined more precisely afterwards. The small amount of cracks that were found do support the conclusion that surface roughening and slip bands are precursors to crack nucleation which corroborates the findings from the first high-power test. The widths of the regions of surface roughening and cracks are given in Table XII.

\section{CONCLUSION}

We have demonstrated that the cyclic stress induced by pulsed heating beyond the yield stress of fully annealed 
OFE copper results in damage to the surface in the form of surface roughening and cracks. The two experiments have shown such damage to occur at $56 \times 10^{6}$ pulses at a calculated $120 \pm 10 \mathrm{~K}$ temperature rise on the surface and at $86 \times 10^{6}$ pulses at a calculated temperature rise of $82 \pm 3 \mathrm{~K}$ on the surface. The measurements of pulsed temperature rise are in reasonable agreement with the calculations for the second high-power test. From the destruction of the high-power coupling aperture, we also know that $56 \times 10^{6}$ pulses at a temperature rise of approximately $250 \mathrm{~K}$ will create enough cracks to cause local melting of the surface. Cracks cause the electrical resistivity of the surface to increase; consequently, the temperature rise increases for the same applied power density.

Since the cavity $Q$ depends on the electrical resistivity of the surface, surface roughening and cracks that ensue will degrade the rf properties of a device long before catastrophic damage such as melting and copper sputtering occur. The numbers given above are not thresholds for damage to occur, rather they are observations of damage for the parameters and under the conditions of these experiments. The number of pulses would correspond to only one or two months of accelerator operation, and, therefore, because of thermal runaway they are pulsed temperature rises that cannot be exceeded when accelerator lifetime is an issue.

These results are for a particular cavity and a particular mode, but they can be applied to OFE copper cavities operating in any standing or traveling wave mode. The cavity geometry can be entered into a field calculating computer program, such as MAFIA or GDFIDL, and the ratio of accelerating gradient to pulsed temperature rise calculated. Our results then give the maximum accelerating gradient if pulsed heating is the limiting phenomenon. It has been observed in recent tests of NLC structures that reducing the pulsed temperature rise in the input power coupler increased the achievable gradient [22].

Preparation of the cavities and, in particular, the end cap surfaces followed standard SLAC procedures for copper to be used in high-power rf applications. It is known that cyclic fatigue is dependent on surface preparations [8], and the light chemical etch performed on the surface for vacuum preparation may have reduced the lifetime. Grain boundaries are primary sights for crack nucleation, and the etching of material within the grain boundaries can increase the stress concentration in that area. There are open questions about whether the gradient limitation from rf pulsed heating can be reduced by different surface preparations, or, alternatively, through the use of dispersion strengthened materials such as GlidCop ${ }^{\circledR}$ [23], operating at lower ambient temperature or diamond coating [24]. For example, it has been shown [4] that the addition of tungsten can increase achievable accelerator gradients by increasing the threshold for damage from rf breakdown.
This experiment shows that pulsed heating is a serious issue with the potential of limiting gradients in future accelerators.

\section{ACKNOWLEDGMENTS}

The authors would like to acknowledge Professor S. Schultz for his suggestion of using a second mode to measure the pulsed temperature rise in the cavity. We would like to thank G. Bowden for his assistance in the mechanical design of the test cavity. We would also especially like to thank G. Caryotakis, A. Vlieks, and A. Menegat for their assistance in the running of these experiments. We appreciate contributions from D. Yu and T. Lee to an earlier experiment.

*Electronic address: pritzkau@bigbearnetworks.com

†Electronic address: siemann@slac.stanford.edu

[1] P. B. Wilson, in Proceedings of the ITP Conference on Future High Energy Colliders (University of California, Santa Barbara, 1996).

[2] R. H. Siemann, in Proceedings of the Symposium on Electron Linear Accelerators: In Honor of Richard B. Neal's 80th Birthday, edited by R. H. Siemann (Stanford Linear Accelerator Center, Stanford, 1997), pp. 45-54 (SLAC Report No. SLAC-R-526), http://www.slac. stanford.edu/pubs/slacreports/slac-r-526.html.

[3] C. Adolphsen, W. Baumgartner, R. K. Jobe, F. L. Pimpec, R. Loewen, D. McCormick, M. C. Ross, T. Smith, J.W. Wang, and T. Higo, in Proceedings of the 2001 Particle Accelerator Conference, Chicago (IEEE, Piscataway, NJ, 2001), pp. 478-480.

[4] H. H. Braun, S. Döbert, I. Syratchev, M. Taborelli, I. Wilson, and W. Wuensch, Technical Report CLIC Note No. 535, European Organization for Nuclear Research - PS Division, 2002.

[5] H. M. Musal, Jr., Laser Induced Damage in Optical Materials, 1979, NBS Special Publication No. 568 (U.S. GPO, Washington, DC, 1980), pp. 159-173.

[6] CRC Handbook of Chemistry and Physics, edited by D. R. Lide (CRC Press Inc., Boca Raton, FL, 2000), 81 st ed.

[7] C. D. Marrs, W. N. Faith, J. H. Dancy, and J. O. Porteus, Appl. Opt. 21, 4063 (1982).

[8] J. A. Bannantine, J. J. Comer, and J. L. Handrock, Fundamentals of Metal Fatigue Analysis (Prentice Hall, Englewood Cliffs, NJ, 1990).

[9] V.F. Kovalenko, Physics of Heat Transfer and Electrovacuum Devices (Sovetskoe Radio, Moscow, 1975).

[10] O. A. Nezhevenko, in Proceedings of the 1997 Particle Accelerator Conference, Vancouver, Canada (IEEE, Piscataway, NJ, 1997), pp. 3013-3014.

[11] D. Roylance, Mechanics of Materials (John Wiley \& Sons, Inc., New York, 1996).

[12] C. GmbH, MAFIA User Guide 4.00, http://www.cst.de, Darmstadt, Germany, 1998. 
[13] W. Bruns, in Proceedings of the 1999 Particle Accelerator Conference, New York (IEEE, Piscataway, NJ, 1999), pp. 2767-2768.

[14] 1,1,1 Trichloroethane is a product of Dow Chemical Co.

[15] Enbond Q527 is a product of Enthone Inc., New Haven, CT.

[16] Oxyban 60 is a product of Thiokol/Dynachem Corp., Tustin, CA.

[17] D. P. Pritzkau, Ph.D. thesis, Stanford University, 2001 (SLAC Report No. SLAC-R-577), http://www.slac. stanford.edu/pubs/slacreports/slac-r-577.html.

[18] Cyclic Deformation and Fatigue of Metals, edited by M. Bílý, Materials Science Monographs Vol. 78 (Elsevier, Amsterdam, 1993).
[19] M. Klesnil and P. Lukáš, in Fatigue of Metallic Materials, Materials Science Monographs Vol. 71 (Elsevier, Amsterdam, 1992).

[20] ANSOFT, Ansoft HFSS Ver 5, http://www.ansoft.com, Pennsylvania, 1998.

[21] R.W. K. Honeycombe, The Plastic Deformation of Metals (Edward Arnold, London, 1984).

[22] D. Burke (private communication).

[23] GlidCop is a registered trademark of the OM Group, Inc., Cleveland, $\mathrm{OH}$.

[24] X. E. Lin, Technical Report No. ARDB-185, Accelerator Research Department B, Stanford Linear Accelerator Center, 1998. 